\title{
35. UNDERWAY GEOPHYSICAL DATA FROM DEEP SEA DRILLING PROJECT LEG 61: NAVIGATION, BATHYMETRY, MAGNETICS, AND SEISMIC PROFILES ${ }^{1}$
}

\author{
Seymour O. Schlanger, Hawaii Institute of Geophysics, Honolulu, Hawaii \\ and \\ Roger L. Larson, Lamont-Doherty Geological Observatory, Palisades, New York
}

\section{INTRODUCTION}

Leg 61, which was devoted to drilling at Site 462 in the Nauru Basin, began at Apra Harbor, Guam, Mariana Islands on May 22, 1979 and ended at Majuro Atoll, Marshall Islands, on July 29, 1979. In the course of the cruise, the Glomar Challenger logged 2395 miles of steaming, during which the following data were collected: bathymetric data for 2015 miles, magnetic data for 2020 miles, and seismic reflection data for 2028 miles. These data were recorded at sea under the direction of Laboratory Officers G. Bode and M. Lehman. Data processing after the cruise was carried out by the Scripps Institution of Oceanography Geologic Data Center. Leg 61 is referenced as Geologic Data Center Cruise I.D. No. 124. The following forms of data are available from the Information Handling Group, Deep Sea Drilling Project, A-031, Scripps Institution of Oceanography, La Jolla, California 92093:

1) Profiles of depths and magnetic anomalies plotted versus distance. Dates (day/month) and positions of major course changes (greater than $30^{\circ}$ ) are annotated. Sections of track having sub-bottom profiler (airgun) records are identified by a solid black line along the bottom of the profile.

2) Navigation listing of times and positions of course and speed changes, fixes, and drift velocity.

3) Depth compilation plots, in meters (assumed sound velocity of $1500 \mathrm{~m} / \mathrm{s}$ ), at approximately 1 -mile spacing, plotted at 4 in./degree, with standard Defense Mapping Agency Hydrographic Center Office B.C. Series boundaries (see index chart).

4) Plots of magnetic anomaly profiles along trackmap scale $=1 / 2$ " $/$ degree; anomaly scale between $15^{\circ} \mathrm{N}$ and $15^{\circ} \mathrm{S}$ latitude $=500$ gamma/inch; anomaly scale north of $15^{\circ} \mathrm{N}$ and south of $15^{\circ} \mathrm{S}=1000$ gamma/inch; from values retrieved at approximately 1 mile spacing and regional field removed using the 1975 IGRF.

5) Card decks of navigation, depth and magnetics.

6) S.I.O. Sample Index-list of the drill sites and beginning and end times and positions of all underway records collected on the cruise leg.

7) Microfilm or Xerox copies of:

a) Echosounder records $(12 \mathrm{kHz}, 3.5 \mathrm{kHz})$

b) Sub-bottom profiler records (airgun)

\footnotetext{
${ }^{1}$ Initial Reports of the Deep Sea Drilling Project, Volume 61.
}

c) Magnetometer records

d) Underway data log

Beginning with Leg 45 (December 1975), depths are recorded on the Glomar Challenger in meters $(1500 \mathrm{~m} / \mathrm{s}$ velocity). Prior to Leg 45 , depths were recorded in fathoms ( $800 \mathrm{fm} / \mathrm{s}$ velocity). Beginning with Leg 54 (June 1977), the magnetic regional field is removed, using the 1975 IGRF.

\section{METHODS}

Navigation. Satellite fixes and course and speed changes were encoded aboard the Challenger from data given in the underway geophysical log. The data were keypunched on shore and put through a navigation smoothing program, edited on the basis of reasonable shipdrift velocities and a deck of corrected navigation points punched out for later merging with the depth and magnetic data. Table 1 contains detailed time, position, satellite-fix, distance, course, speed, and drift data. The ship's track, with day and hour ticks in GMT, is shown as Figure 1.

Depth. The depths scaled from echo sounders $(1500 \mathrm{~m} / \mathrm{s}$ calibrated sound velocity) were recorded at sea in the underway geophysical log book at 5-minute intervals. The depths were keypunched on shore and edited in the same fashion as the magnetics. The bathymetric profiles obtained are shown on Figure 2 where they are keyed to both nautical miles (n.m.) from Guam and the day and hour time ticks shown on Figure 1.

Magnetics. The magnetics scaled from analog records produced on the Geometrics Magnetometer were recorded at sea in the underway geophysical log book in gammas at 5-minute intervals. The magnetics were keypunched on shore, put through a profile program, and edited by comparison to the original analog records. These magnetic profiles are shown in Figure 2, where they are keyed to both n.m. from Guam and the day and hour ticks shown on Figure 1.

Seismic Profiles. The energy sources were Bolt air guns. Generally 120 - and 80 -in. ${ }^{3}$ guns were streamed as a pair. Occasionally, one or both of these were pulled for repairs, and 20 - and 40 -in. ${ }^{3}$ guns used as replacements. Returns were recorded on 2 EPC recorders. Number 1 (EDO \#1 on the records) was set for a 10-second sweep with the bandpass filter set at the 20 -to- $80-\mathrm{Hz}$ range. Recorder number 2 (EDO \#2 on the records) was set for a 5-second sweep, with a trigger delay of 2 to 5 seconds, depending on the water depth, and a band-pass filter setting of 40 to $160 \mathrm{~Hz}$. Photographs of the records are reproduced in this chapter as Figure $3 \mathrm{~A}$ to $3 \mathrm{JJ}$, keyed to the bathymetric, magnetic, time, and distance data shown in Figure 2. Figures $3 \mathrm{~A}$ to $3 \mathrm{JJ}$ have been annotated to point up particular geologic features. Each photograph therefore can be located along the Leg 61 track shown in Figure 1.

\section{RESULTS AND DISCUSSION}

Glomar Challenger left Apra Harbor on the west coast of Guam and steered south to clear Cocos Island at the south tip of Guam. The southern third of Guam is a remnant of a volcanic caldera of Miocene age, the western half of which was down-faulted and now lies below sea level (Tracey et al., 1964). The bathymetry 
Table 1. Navigation data for Leg 61 .

\begin{tabular}{|c|c|c|c|c|c|c|c|c|c|c|}
\hline & & & & LATI & TUDE & LONG & ITUDE & & & CTUA \\
\hline DA & MO & YR & TIME & DEG & MIN & DEG & MIN & DIST & SPEED & CSE \\
\hline 22 & 5 & 1978 & - 350 & 13 & 26.00 & 144 & 34.50 & 0.0 & 4.1 & 155 \\
\hline 22 & 5 & $\begin{array}{l}1978 \\
1978\end{array}$ & $\begin{array}{l}357 \\
46\end{array}$ & 13 & $\begin{array}{l}25.6 \\
24.7\end{array}$ & 14 & 3 & 0.5 & $\begin{array}{l}6.5 \\
6.5\end{array}$ & $\begin{array}{l}157 \\
180\end{array}$ \\
\hline $\begin{array}{l}22 \\
22\end{array}$ & $\begin{array}{l}5 \\
5\end{array}$ & $\begin{array}{l}1978 \\
1978\end{array}$ & $\begin{array}{l}46 \\
452\end{array}$ & $\begin{array}{l}13 \\
13\end{array}$ & $\begin{array}{l}24.7 \\
19.7\end{array}$ & $\begin{array}{l}144 \\
144\end{array}$ & $\begin{array}{l}35.1 \\
35.1\end{array}$ & $\begin{array}{l}1.5 \\
6.4\end{array}$ & $\begin{array}{l}6.5 \\
8.7\end{array}$ & $\begin{array}{l}180 \\
180\end{array}$ \\
\hline 22 & 5 & 1978 & - 58 & 13 & 17.40 & 144 & 35.10 & 8.7 & 9.7 & 184 \\
\hline 22 & 5 & 1978 & 550 & 13 & 10.6 & 144 & 34.6 & 15.5 & 8.9 & 105 \\
\hline 22 & 5 & 1978 & $\begin{array}{l}\cdot 622 \\
<s\end{array}$ & 13 & 9.40 & 144 & 39.30 & 20.3 & 8.0 & 105 \\
\hline 22 & 5 & 1978 & $\begin{array}{r}658 \\
.86\end{array}$ & 13 & 8.1 & 144 & 44.1 & $\begin{array}{l}25.1 \\
34.2\end{array}$ & $\begin{array}{l}8.0 \\
8.3\end{array}$ & 104 \\
\hline $\begin{array}{l}22 \\
22\end{array}$ & $\begin{array}{l}5 \\
5\end{array}$ & $\begin{array}{l}1978 \\
1978\end{array}$ & $\begin{array}{l}886 \\
.1236\end{array}$ & $\begin{array}{l}13 \\
12\end{array}$ & $\begin{array}{r}5.90 \\
58.60\end{array}$ & $\begin{array}{l}144 \\
145\end{array}$ & $\begin{array}{l}53.10 \\
30.60\end{array}$ & $\begin{array}{l}34.2 \\
71.4\end{array}$ & $\begin{array}{l}8.3 \\
8.2\end{array}$ & $\begin{array}{l}101 \\
103\end{array}$ \\
\hline 22 & 5 & 1978 & 140 & 12 & 56.0 & 145 & 42.1 & 82.9 & 8.4 & 103 \\
\hline 22 & 5 & 1978 & $\cdot 1554$ & 12 & 52.40 & 145 & 58.00 & 98.8 & 8.4 & 101 \\
\hline 22 & 5 & 1978 & $\cdot 1616$ & 12 & 51.80 & 146 & 1.10 & 101.9 & 8.2 & 103 \\
\hline 23 & 5 & 1978 & 00 & 12 & 37.1 & 147 & 4.2 & 165.2 & 8.2 & 103 \\
\hline 23 & 5 & 1978 & 018 & 12 & 36.50 & 147 & 6.60 & 167.6 & 8.6 & 102 \\
\hline 23 & 5 & 1978 & $\cdot 418$ & 12 & $\begin{array}{r}29.40 \\
27\end{array}$ & $\begin{array}{l}147 \\
147\end{array}$ & $\begin{array}{l}40.90 \\
56.10\end{array}$ & $\begin{array}{l}201.8 \\
216.9\end{array}$ & $\begin{array}{l}8.2 \\
84\end{array}$ & 98 \\
\hline 23 & 5 & 1978 & $\begin{array}{r}: 68 \\
: 1388\end{array}$ & 12 & $\begin{array}{l}27.20 \\
15.80\end{array}$ & $\begin{array}{l}147 \\
148\end{array}$ & $\begin{array}{l}56.10 \\
58.20\end{array}$ & $\begin{array}{l}2168.9 \\
278.6\end{array}$ & 8.3 & 101 \\
\hline 23 & 5 & $\begin{array}{l}1978 \\
1978\end{array}$ & $\begin{array}{r}1328 \\
1538\end{array}$ & 12 & $\begin{array}{l}15.80 \\
12.2\end{array}$ & $\begin{array}{l}148 \\
149\end{array}$ & 16.3 & 296.6 & $\begin{array}{l}8.3 \\
8.5\end{array}$ & $\begin{array}{l}102 \\
134\end{array}$ \\
\hline 23 & 5 & $\begin{array}{l}1978 \\
1978\end{array}$ & $\begin{array}{l}1538 \\
1558\end{array}$ & 12 & $\begin{array}{l}12.2 \\
10.2\end{array}$ & 149 & 18.3 & 299.4 & $\begin{array}{l}8.5 \\
8.3\end{array}$ & $\begin{array}{l}134 \\
102\end{array}$ \\
\hline 23 & $\begin{array}{l}5 \\
5\end{array}$ & $\begin{array}{l}1978 \\
1978\end{array}$ & $\begin{array}{r}1558 \\
.162\end{array}$ & 12 & $\begin{array}{l}10.2 \\
10.10\end{array}$ & $\begin{array}{l}149 \\
149\end{array}$ & 18.90 & 300.0 & 8.2 & $\begin{array}{l}102 \\
102\end{array}$ \\
\hline 23 & $\begin{array}{l}5 \\
5\end{array}$ & 1978 & $\begin{array}{r}* 162 \\
: 1818\end{array}$ & 12 & $\begin{array}{r}10.10 \\
6.20\end{array}$ & 149 & 37.40 & 318.5 & 8.7 & \\
\hline $\begin{array}{l}23 \\
23\end{array}$ & $\begin{array}{l}5 \\
5\end{array}$ & 1978 & $\begin{array}{r}\cdot 1818 \\
\cdot 204\end{array}$ & 12 & $\begin{array}{l}6.20 \\
3.10\end{array}$ & 149 & 50.90 & 332.0 & 7.8 & 104 \\
\hline $\begin{array}{l}23 \\
23\end{array}$ & 5 & 1978 & $\begin{array}{r}204 \\
2348\end{array}$ & 12 & $\begin{array}{r}3.10 \\
50.1\end{array}$ & 150 & 19.7 & 361.1 & 7.8 & 106 \\
\hline $\begin{array}{l}23 \\
24\end{array}$ & 5 & 1978 & $\begin{array}{r}2348 \\
00\end{array}$ & 11 & $\begin{array}{l}56.1 \\
55.7\end{array}$ & 15 & 21.3 & 362.7 & 7.8 & 106 \\
\hline 24 & 5 & 1978 & - 110 & 11 & 53.10 & is & 30.20 & 371.8 & 7.8 & 102 \\
\hline 24 & 5 & 1978 & 131 & 11 & 52.5 & 150 & 32.9 & 374.5 & 7.8 & 101 \\
\hline 24 & 5 & 1978 & - 228 & 11 & 51.10 & 150 & 40.40 & 382.0 & 7.9 & 101 \\
\hline 24 & 5 & 1978 & - 256 & 11 & 50.40 & 150 & 44.10 & 385.6 & 7.5 & 98 \\
\hline 24 & 5 & 1978 & $: 330$ & II & $\begin{array}{l}49.80 \\
48.80\end{array}$ & 150 & & 389.9 & 7.1 & 101 \\
\hline 24 & 5 & 1978 & $: \begin{array}{l}446 \\
518\end{array}$ & II & $\begin{array}{l}48.80 \\
47.40\end{array}$ & $\begin{array}{l}150 \\
150\end{array}$ & 53.90 & 395.4 & 7.8 & 100 \\
\hline 24 & 5 & $\begin{array}{l}1978 \\
1978\end{array}$ & $\begin{array}{r}518 \\
543\end{array}$ & $\begin{array}{l}11 \\
11\end{array}$ & & 151 & 2.00 & 403.4 & 7. & 101 \\
\hline 24 & 5 & $\begin{array}{l}1978 \\
1978\end{array}$ & $\begin{array}{r}.543 \\
.552\end{array}$ & ${ }_{11}^{11}$ & $\begin{array}{l}46.8 \\
46.50\end{array}$ & $\begin{array}{l}151 \\
151\end{array}$ & $\begin{array}{l}5.2 \\
6.40\end{array}$ & $\begin{array}{l}406.7 \\
407.8\end{array}$ & 7. & 10 \\
\hline 24 & 5 & $\begin{array}{l}1978 \\
1978\end{array}$ & $\begin{array}{r}.532 \\
738\end{array}$ & 11 & $\begin{array}{l}4 \\
4\end{array}$ & 11 & $\begin{array}{r}6.40 \\
19.80\end{array}$ & $\begin{array}{l}407.8 \\
421.3\end{array}$ & 7 & 103 \\
\hline 24 & 5 & $\begin{array}{l}1978 \\
1978\end{array}$ & $\begin{array}{l}138 \\
1358\end{array}$ & 11 & 30 & 1. & 7 & $\begin{array}{l}421.3 \\
469.6\end{array}$ & $\begin{array}{l}7.6 \\
6.4\end{array}$ & 105 \\
\hline 24 & $\begin{array}{l}5 \\
5\end{array}$ & $\begin{array}{l}1978 \\
1978\end{array}$ & $\begin{array}{r}14310 \\
.140\end{array}$ & II & 30.20 & 15 & 8.60 & 470.9 & $\begin{array}{l}6.4 \\
7.3\end{array}$ & 106 \\
\hline $\begin{array}{l}24 \\
24\end{array}$ & $\begin{array}{l}5 \\
5\end{array}$ & $\begin{array}{l}1978 \\
1978\end{array}$ & 1421 & 11 & 29 & is & 9.9 & 472.2 & $\begin{array}{l}7.3 \\
8.5\end{array}$ & $\begin{array}{l}104 \\
104\end{array}$ \\
\hline 24 & 5 & 1978 & $\cdot 1438$ & 11 & 29.30 & is & 12.30 & 474.6 & 8.1 & $\begin{array}{l}104 \\
110\end{array}$ \\
\hline 24 & 5 & 1978 & 1443 & ii & 29.1 & is & 12.9 & 475.3 & 8.1 & 107 \\
\hline 24 & 5 & 1978 & $\cdot 1558$ & 11 & 26.10 & is & 22.80 & 485.4 & 8. & 105 \\
\hline 24 & 5 & 1978 & $\bullet 170$ & 11 & 23.90 & 152 & 31.10 & 493. & 8. & 105 \\
\hline 25 & 5 & & 00 & 11 & 9.2 & 15 & 27. & 550 & 8 & 105 \\
\hline 25 & 5 & 1978 & $\begin{array}{r}* \\
{ }^{2}\end{array}$ & 11 & 8.80 & 15 & 28.30 & 551 & 8. & 101 \\
\hline 25 & 5 & $\begin{array}{l}1978 \\
1978\end{array}$ & $\begin{array}{r}034 \\
-324\end{array}$ & ${ }_{i i}^{11}$ & $\begin{array}{l}8.2 \\
4.50\end{array}$ & 153 & 31.6 & 555.3 & 8.4 & 99 \\
\hline $\begin{array}{l}25 \\
25\end{array}$ & $\begin{array}{l}5 \\
5\end{array}$ & $\begin{array}{l}1978 \\
1978\end{array}$ & $\begin{array}{r}324 \\
352\end{array}$ & i1 & & $\begin{array}{l}153 \\
153\end{array}$ & & $\begin{array}{l}579.1 \\
583.2\end{array}$ & 8.9 & $\begin{array}{r}98 \\
100\end{array}$ \\
\hline 25 & 5 & & $\cdot 428$ & II & & is & & 58 & 9.0 & ic \\
\hline 25 & 5 & 1978 & . 630 & io & 59. & is & 23.60 & 607.0 & 8.8 & 100 \\
\hline 25 & 5 & 1978 & $\cdot 1350$ & 10 & 49.00 & is & 28.50 & 671.6 & 8 & 103 \\
\hline 25 & 5 & 1978 & .156 & 10 & 46.7 & 155 & 38.50 & 681. & 8. & 103 \\
\hline 25 & 5 & 1978 & $\bullet 153$ & 10 & 45. & is & 43.00 & 686 & 8. & 10 \\
\hline 25 & 5 & 1978 & $* 1750$ & 10 & 40. & 15 & 0 . & 704 & 8. & 10 \\
\hline 25 & 5 & 1978 & •1936 & (1) & 37. & 15 & 15. & 719 & & 10 \\
\hline 25 & 5 & 1978 & 213 & ) & 33 & 15 & 31. & 735 & & ic \\
\hline 25 & 5 & 1978 & $\cdot 2316$ & & 30 & 15 & 43. & 747 & r. & it \\
\hline 26 & 5 & 197 & 00 & . & 29 & 15 & 49. & 75 & 7 & ic \\
\hline 26 & 5 & 197 & - 14 & (1) & 27. & 15 & 57 & 76 & & 10 \\
\hline 26 & 5 & 19 & 13 & If & & 15 & 0. & & & 10 \\
\hline 26 & 5 & 197 & $\cdot 3$ & 10 & 23 & 15 & 14. & 77 & & 10 \\
\hline 26 & 5 & 19 & $\cdot 34$ & I & & 15 & 19 & & & 0 \\
\hline 26 & 5 & 197 & - 71 & 1 & 18.3 & 1. & 48 & & & 10 \\
\hline 26 & 5 & 197 & 76 & & 17. & 1 & 54 & & & 16 \\
\hline $2 t$ & 5 & 19 & 15 & & 4. & 1 & 59 & & & 16 \\
\hline $2 t$ & 5 & 19 & $\cdot 15$ & 10 & 3. & 1 & 1. & & & is \\
\hline 26 & 5 & 19 & $\cdot 1$ & 0 & 54. & 1. & 28. & & & 16 \\
\hline 26 & 5 & 19 & 18 & 9 & & 1 & 28 & & & 11 \\
\hline 27 & 5 & 19 & $\Omega$ & 9 & & & 1 & & & \\
\hline 27 & 5 & 19 & 1 & 9 & & & & & 8 & 18 \\
\hline 27 & 5 & 19 & - 14 & 0 & & & & & & 1 \\
\hline 27 & 5 & 19 & 2 & 9 & & & & & & Ic \\
\hline 27 & 5 & 15 & 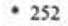 & 9 & & & & & & If \\
\hline 27 & 5 & 19 & $\cdot 3$ & 9 & & & 41 & & & 1 \\
\hline 27 & 5 & 19 & $\cdot 4$ & 9 & & I & 51 & & & 11 \\
\hline 27 & 5 & 19 & * 6 & 5 & 2 & It & 2 & & & 18 \\
\hline 27 & 5 & 15 & 6 & & & 1 & 9 & & & 16 \\
\hline 27 & 5 & 19 & $\bullet 11$ & & & 1 & 50 & & & 10 \\
\hline 27 & 5 & 19 & 12 & & & & 56 & & 9 & 9 \\
\hline 27 & 5 & 15 & $\cdot 13$ & & & & 12 & & & 9 \\
\hline 27 & 5 & 15 & $\cdot 14$ & & 9. & & 17. & & & 9 \\
\hline 27 & 5 & 15 & $\bullet 1$ & & 9. & I & 22 & & & 9 \\
\hline 27 & 5 & 15 & $*_{1}$ & 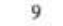 & 8. & 1 & 33. & & & 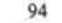 \\
\hline 27 & $s$ & 1 & 15 & & 6 & & 0 & & & \\
\hline & 5 & & 22 & 1 & & & 1 & & & \\
\hline 28 & 5 & & 0 & $\varepsilon$ & & & 24 & & & \\
\hline 28 & 5 & & 0 & & & & 24 & & & \\
\hline 28 & 5 & 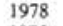 & $\cdot 05$ & 8 & 26. & 16 & 3 & & & 12 \\
\hline 28 & 5 & 197 & 11 & & 25. & 16 & 3. & 118. & & \\
\hline 28 & 5 & & * & 8 & 24. & 16 & 34. & & 9. & 11 \\
\hline 28 & 5 & 19 & 15 & 8 & 22 & 16 & 3 & & 9.3 & 11 \\
\hline 28 & 5 & 1978 & $\cdot 236$ & 8 & 19.30 & 163 & 44.30 & 1195.4 & & 1177 \\
\hline
\end{tabular}


Table 1. (Continued).

\begin{tabular}{|c|c|c|c|}
\hline & & & \\
\hline DA & MO & YR & TIME \\
\hline 28 & 5 & 1978 & $\cdot 314$ \\
\hline 28 & 5 & 1978 & - 348 \\
\hline 28 & 5 & 1978 & 50 \\
\hline 28 & 5 & 1978 & .640 \\
\hline 28 & 5 & 1978 & 710 \\
\hline 28 & 5 & 1978 & 842 \\
\hline 28 & 5 & 1978 & 1025 \\
\hline 28 & 5 & 1978 & 115 \\
\hline 28 & 5 & 1978 & iiii \\
\hline 28 & 5 & 1978 & 1150 \\
\hline 28 & 5 & 1978 & $\cdot 1226$ \\
\hline 28 & 5 & 1978 & $\cdot 1312$ \\
\hline 28 & 5 & 1978 & 1330 \\
\hline 28 & 5 & 1978 & 143 \\
\hline 28 & 5 & 1978 & $\cdot 1416$ \\
\hline 28 & 5 & 1978 & 1434 \\
\hline 28 & 5 & 1978 & $\cdot 150$ \\
\hline 28 & 5 & 1978 & 1512 \\
\hline 28 & 5 & 1978 & 1516 \\
\hline 28 & 5 & 1978 & $\cdot 1550$ \\
\hline 28 & 5 & 1978 & 1550 \\
\hline 7 & 6 & 1978 & $\cdot 220$ \\
\hline 9 & 6 & 1978 & .35 \\
\hline 8 & 7 & 1978 & $\cdot 1850$ \\
\hline 8 & 7 & 1978 & 1850 \\
\hline 8 & 7 & 1978 & 1924 \\
\hline 8 & 7 & 1978 & $\cdot 1958$ \\
\hline 8 & 7 & 1978 & $\cdot 2226$ \\
\hline 8 & 7 & 1978 & $\cdot 2348$ \\
\hline 9 & $?$ & 1978 & 00 \\
\hline 9 & 7 & 1978 & - 140 \\
\hline 9 & & 1978 & $\cdot 340$ \\
\hline 9 & $?$ & 1978 & 40 \\
\hline 9 & 7 & 1978 & - 912 \\
\hline 9 & 7 & 1978 & $\cdot 106$ \\
\hline 9 & 7 & 1978 & 1125 \\
\hline 9 & 7 & 1978 & $\cdot 1132$ \\
\hline 9 & 7 & 1978 & 1138 \\
\hline 9 & 7 & 1978 & $\cdot 1152$ \\
\hline 9 & 7 & 1978 & $\cdot 152$ \\
\hline 9 & 7 & 1978 & 1745 \\
\hline 9 & 7 & 1978 & $\cdot 2048$ \\
\hline 9 & 7 & 1978 & 2121 \\
\hline 9 & 7 & 1978 & $\cdot 2136$ \\
\hline 9 & 7 & 1978 & 2242 \\
\hline 9 & 7 & 1978 & .2250 \\
\hline 9 & 7 & 1978 & 2250 \\
\hline 14 & 7 & 1978 & 2131 \\
\hline 14 & 7 & 1978 & .2134 \\
\hline 14 & 7 & 1978 & 2141 \\
\hline 14 & 7 & 1978 & .228 \\
\hline 14 & 7 & 1978 & $\cdot 2230$ \\
\hline 15 & 7 & 1978 & 00 \\
\hline is & 7 & 1978 & . 014 \\
\hline 15 & 7 & 1978 & 32 \\
\hline 15 & 7 & 1978 & $\begin{array}{r}32 \\
-714\end{array}$ \\
\hline 15 & 7 & 1978 & • 11132 \\
\hline is & 7 & 1978 & 122 \\
\hline 15 & 7 & 1978 & 1435 \\
\hline 15 & 7 & 1978 & $\begin{array}{r}\cdot 1524 \\
\end{array}$ \\
\hline is & 7 & 1978 & 1637 \\
\hline is & 7 & 1978 & 1839 \\
\hline 15 & 7 & 1978 & $\bullet 1852$ \\
\hline 15 & 7 & 1978 & $\cdot 2038$ \\
\hline is & 7 & 1978 & $\cdot 2144$ \\
\hline 15 & 7 & 1978 & .234 \\
\hline is & 7 & 1978 & $\cdot 2326$ \\
\hline 16 & 7 & 1978 & 00 \\
\hline 16 & 7 & 1978 & - 262 \\
\hline 16 & 7 & 1978 & .84 \\
\hline 16 & 7 & 1978 & 8 \\
\hline 16 & $?$ & 1978 & 12 \\
\hline 16 & 7 & 1978 & 1221 \\
\hline 16 & 7 & 1978 & $\cdot 1$ \\
\hline 16 & 7 & 1978 & 1236 \\
\hline 27 & 7 & 1978 & .18 \\
\hline 27 & 7 & 1978 & 52 \\
\hline 27 & 7 & 1978 & 53 \\
\hline 27 & 7 & 1978 & 630 \\
\hline 27 & 7 & 1978 & - 650 \\
\hline 27 & 7 & 197 & - 836 \\
\hline 27 & 7 & 1978 & 90 \\
\hline 27 & 7 & 1978 & $\cdot 14$ \\
\hline 27 & 7 & & $\cdot 182$ \\
\hline 28 & 7 & 19 & $\cdot 146$ \\
\hline 28 & 7 & 19 & - 74 \\
\hline 28 & 7 & 19 & 8 \\
\hline 28 & 7 & 19 & \\
\hline 28 & 7 & 19 & $\cdot 10$ \\
\hline 28 & 7 & 19 & ${ }^{\circ} 1$ \\
\hline 28 & 7 & 197 & ${ }^{2}$ \\
\hline 28 & 7 & 1978 & \\
\hline 28 & 7 & 1978 & $\cdot 2334$ \\
\hline
\end{tabular}

Notes: $\cdot$ Time $=$ GMT: Hours and minutes. Distance $=$ nautical miles. Speed $=$ knots, Course $=$ azimuth $\left(0-360^{\circ}\right)$, DA $=$ day, MO $=$ month. DEG $=$ degree. MIN $=$ minutes. DIST $=$ distance, $\mathrm{CSE}=$ course. $\mathrm{HED}=$ heading (azimuth). $\mathrm{COMNT}=$ comment. $\mathrm{SATL}=$ satellite. $\mathrm{C} / \mathrm{C}=$ course change. $\mathrm{C} / \mathrm{S}=$ speed change. $\mathrm{U} / \mathrm{W}=$ under way. $\mathrm{DEP}=$ depart, 


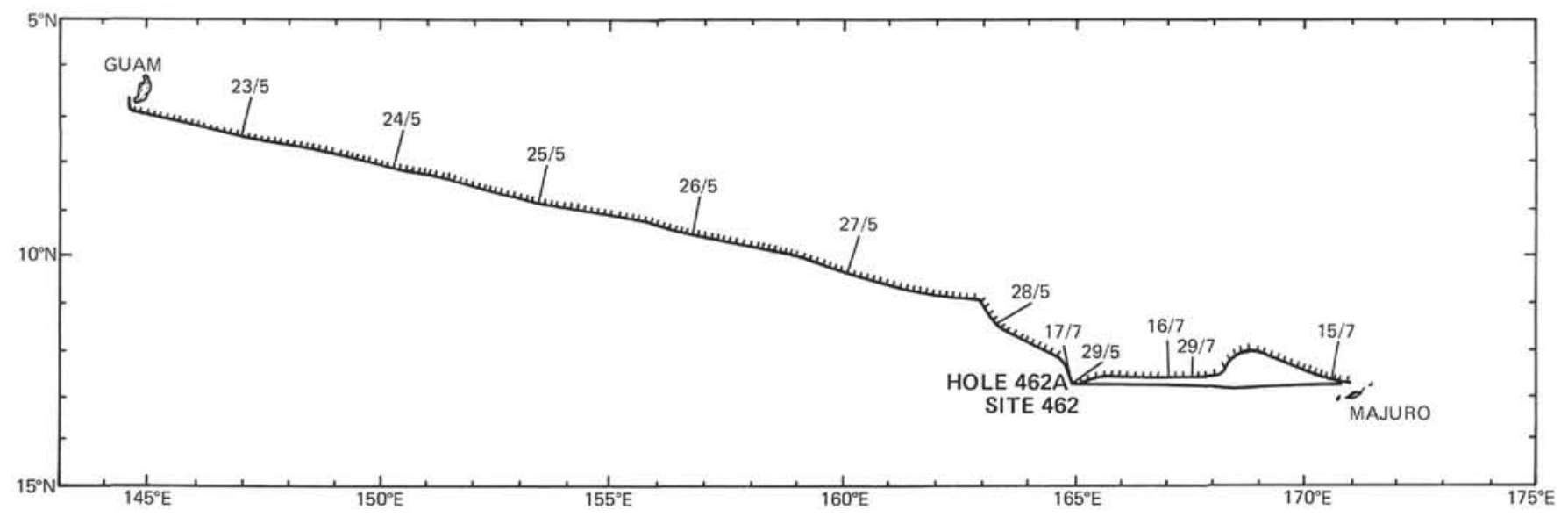

Figure 1. Track of Glomar Challenger on Leg 61: Guam to Site 462, Site 462 to Majuro and return, and Site 462 to Majuro. Hour and day/month time ticks shown are in GMT, e.g., 23/5 is 000023 May GMT position. See Table 1 for details.

(Fig. 3A, 0430Z-0545Z) can be interpreted as a downfaulted volcanic cone. After a course change to $103^{\circ}$ to make for the Nauru Basin, we crossed the Mariana Ridge, the inner trench wall, and the Mariana Trench proper, where a maximum depth of 9750 meters (uncorrected) was recorded (Fig. 3B, 1630Z). Between the outer trench wall and the Mariana Basin, we passed a cluster of seamounts, just starting their journey into the Mariana Trench, the easternmost of which is capped by a drowned reef overlain by pelagic sediments (Fig. 3D). The uppermost reflector taken to be from the reef wall (0615Z) is 2.65 seconds two-way travel time below sea level, a depth of 1990 meters (uncorrected). The region around Enewetak and Bikini to the north has subsided approximately 1600 meters since $70 \mathrm{~m}$.y. ago (Schlanger and Premoli-Silva, this volume). The drowned reef therefore could be of Late Cretaceous age.

West of this reef-capped guyot, the Mariana Basin proper is a relatively featureless plain with a maximum depth along the Leg 61 track of 5890 meters (uncorrected), as seen in Figure 3G. Only a few small volcanic hills project above the sediment cover and a few small diapir structures are present (Fig. 3F). The magnetic profile is also very flat at about the 0 -gamma level. It is difficult to pick any sharp seismic horizon as volcanic basement, but at $1630 \mathrm{Z}, 24 / 5$, the section resembles that drilled at Site 462 (Fig. 3X); a series of widely and equally spaced reflectors at 8.25 -second two-way travel time, below 0.45 seconds of presumed sediment, are acoustically similar to the top of the sill complex at Site 462. At DSDP Site 61 , drilled at $12^{\circ} 05.8^{\prime} \mathrm{N}, 147^{\circ}$ $03.9^{\prime} \mathrm{E}$, at the western extremity of the Mariana Basin (Winterer and Riedel et al., 1971), Upper Cretaceous mudstones lie above amygdaloidal basalt, within which the drill stopped. Reflectors below this "basement" suggested that up to 100 meters of sediment might exist below the basalt. It appears that a sill-and-flow complex similar to but thinner than that in the Nauru Basin may also exist in the Mariana Basin.

The eastern boundary of the Mariana Basin is formed by a group of seamounts (Fig. 3N). Between these sea- mounts and the western Marshall Islands, a semi-transparent sediment layer with bathymetric relief lies above a flatter set of reflectors (Fig. 3O). The uppermost layer probably is a turbidite sequence molded by bottom currents.

Before reaching the Nauru Basin proper, we passed over seamounts in the western Marshalls southwest of Ujelang Atoll (Figs. 3R, S); typical moating can be seen where sediments around seamounts have been eroded. We also passed over Heezen Guyot, because we had picked this guyot as a possible alternate site if time were available after drilling at Site 462 . Heezen Guyot appears to lack a well-defined reef cap, but does have a well-developed pelagic cap (Fig. 3U). The guyot also has a marked magnetic signature (Fig. 2). The Nauru Basin proper is a flat plain, with a depth of about 5180 meters (uncorrected), upon which can be seen turbidite channels and levees (Figure 3W) (see site survey chapter, this volume). Site 462 , in the center of the Nauru Basin, was occupied at 1550Z, 28/5 (Fig. 3X). The site was located on anomaly M-26 (Fig. 2) of Larson and Hilde (1975) (see site chapter, this volume).

After drilling operations at Site 462 , we steamed to Majuro Atoll for a port stop and crew change. Figures $3 \mathrm{Y}$ to $3 \mathrm{CC}$ show the progressively thinning sedimentary layers as the Glomar Challenger approached Majuro.

Upon leaving Majuro Atoll to return to Site 462, we decided to head west-northwest and pass between Namu and Ailinglapalap Atolls, in order to gain additional seismic information on the Marshalls area and the eastern Nauru Basin (Fig. 1). The sedimentary apron on the Marshalls reaches thicknesses up to 1-second two-way travel time (Figure 3EE), similar to those seen around the Line Islands drilled on Leg 33 (Schlanger, Jackson, et al., 1976). The northeast edge of the Nauru Basin (Figs. 3HH and 3II) has a fairly complex acoustic stratigraphy. Rapid lateral changes in reflector thicknesses are seen, and small diapir structures are present (2215Z, 15/7 on Fig. 3HH). At 0630Z, 16/7 (Fig. 3II), there appears to be a boundary between the northeast Nauru Basin and what we might refer to as the central 
Nauru Basin. Northeast of the small volcanic hill that marks the boundary, the sedimentary cover shows irregular reflectors. Between this hill and Site 462 (Fig. $3 \mathrm{JJ})$, the reflectors above the sill complex are flat, but appear to pinch out to the east against a shallowing basement.

Following completion of the renewed drilling at Site 462, Glomar Challenger proceeded directly to Majuro Atoll over the same route we had taken previously.

\section{REFERENCES}

Larson, R. L., and Hilde, T. W. C., 1975. A revised time scale of magnetic reversals for the Early Cretaceous and Late Jurassic. J. Geophys. Res., 80:2586-2594.

Schlanger, S. O., Jackson, E. D., et al., 1976. Init. Repts. DSDP, 33; Washington (U.S. Govt. Printing Office).

Tracey, J. I., Jr., Schlanger, S. O., Stark, J. T., et al., 1964. General geology of Guam. U.S. Geol. Survey Prof. Paper 403A: A1-A104.

Winterer, E. L., Riedel, W. R., et al., 1971. Init. Repts. DSDP, 7: Washington (U.S. Govt. Printing Office), 27-49. 

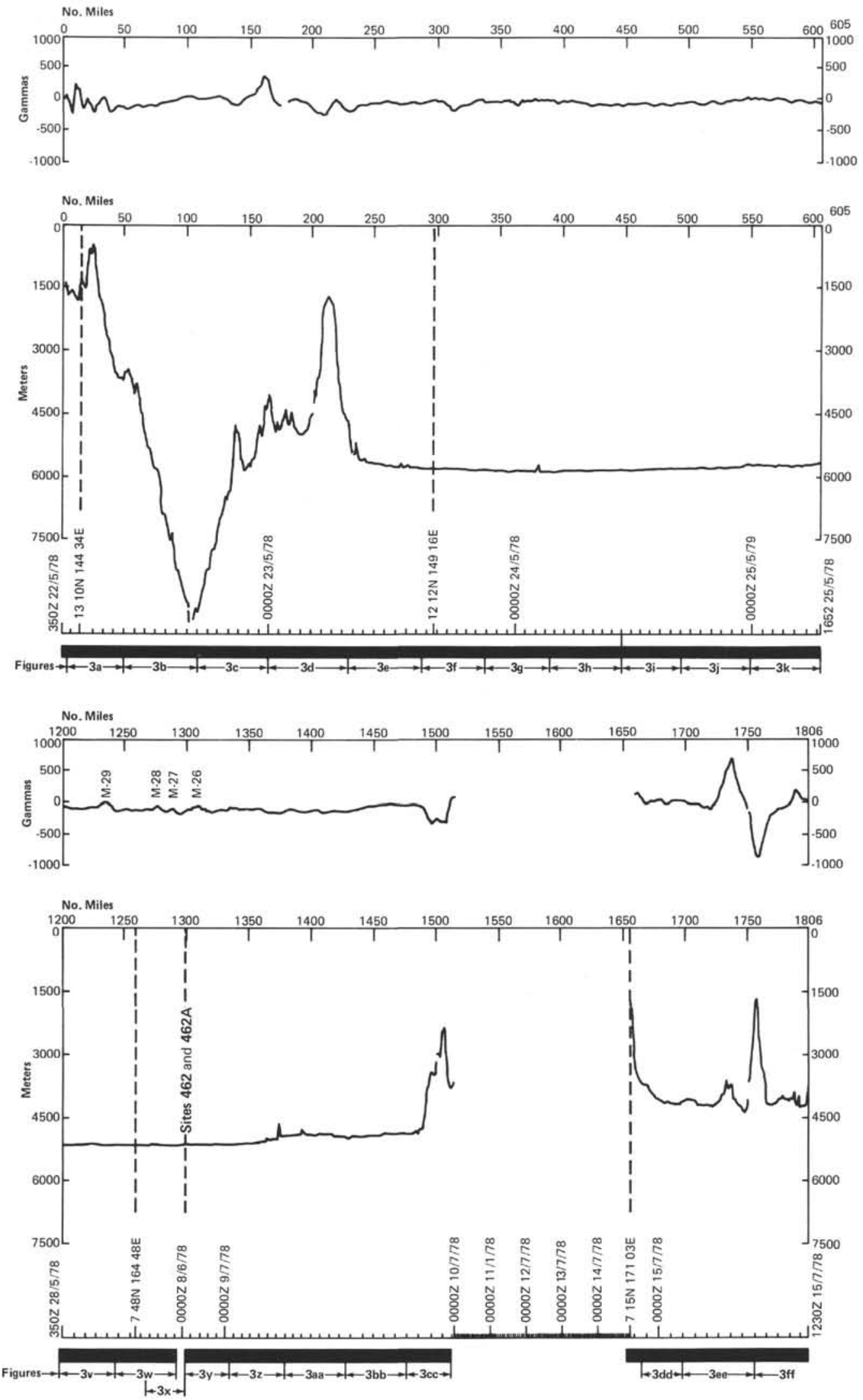

Figure 2. Bathymetry (in meters), magnetic (in gammas) along Glomar Challenger track. Keyed to hour and day/month ticks shown on Figure 1, and to nautical miles from Guam. Shown along the solid black bar are the locations of seismic profiles reproduced as Figures $3 \mathrm{~A}$ to $3 \mathrm{JJ}$. 

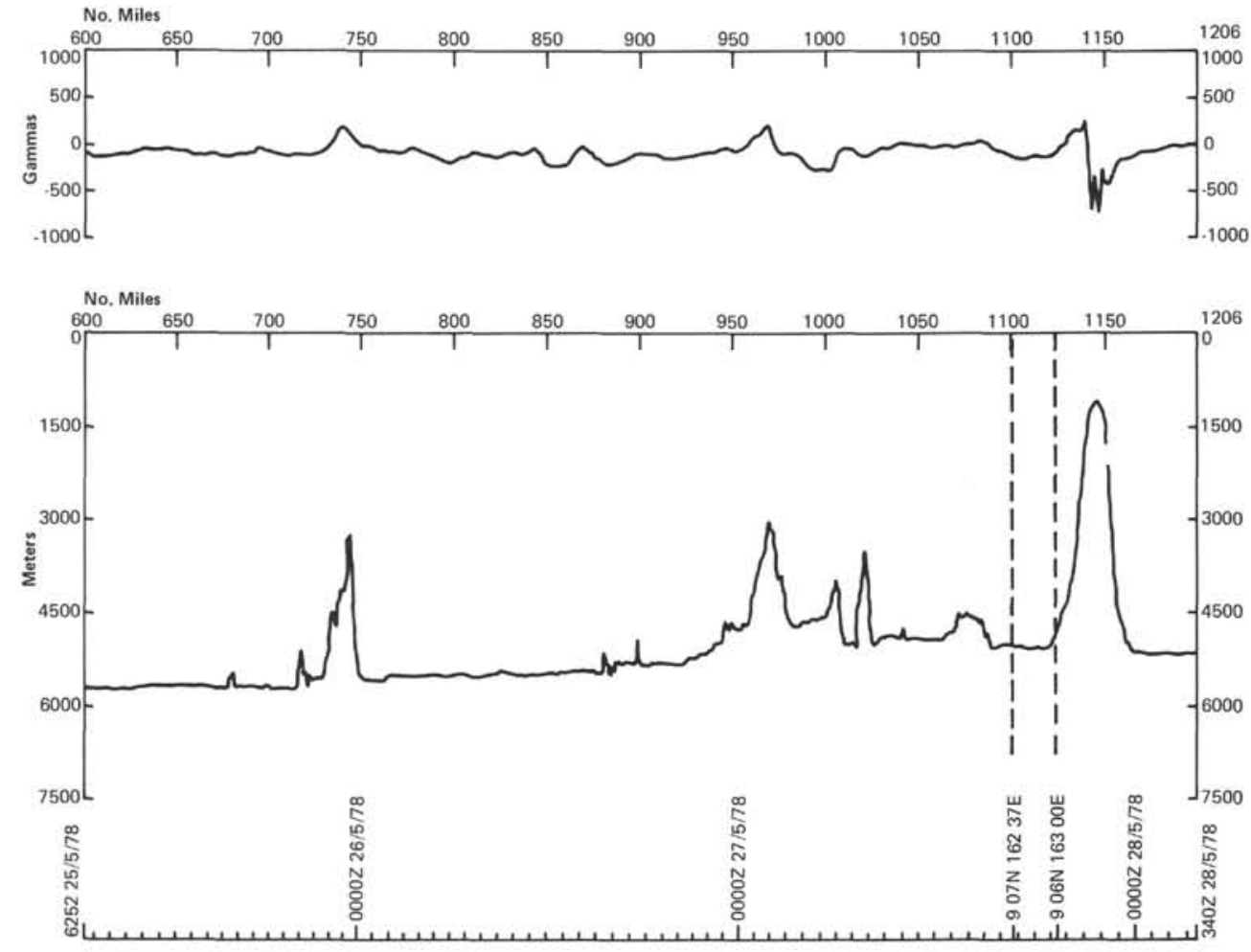

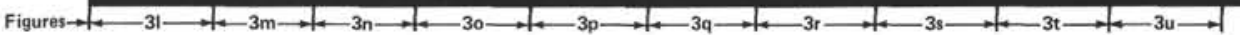
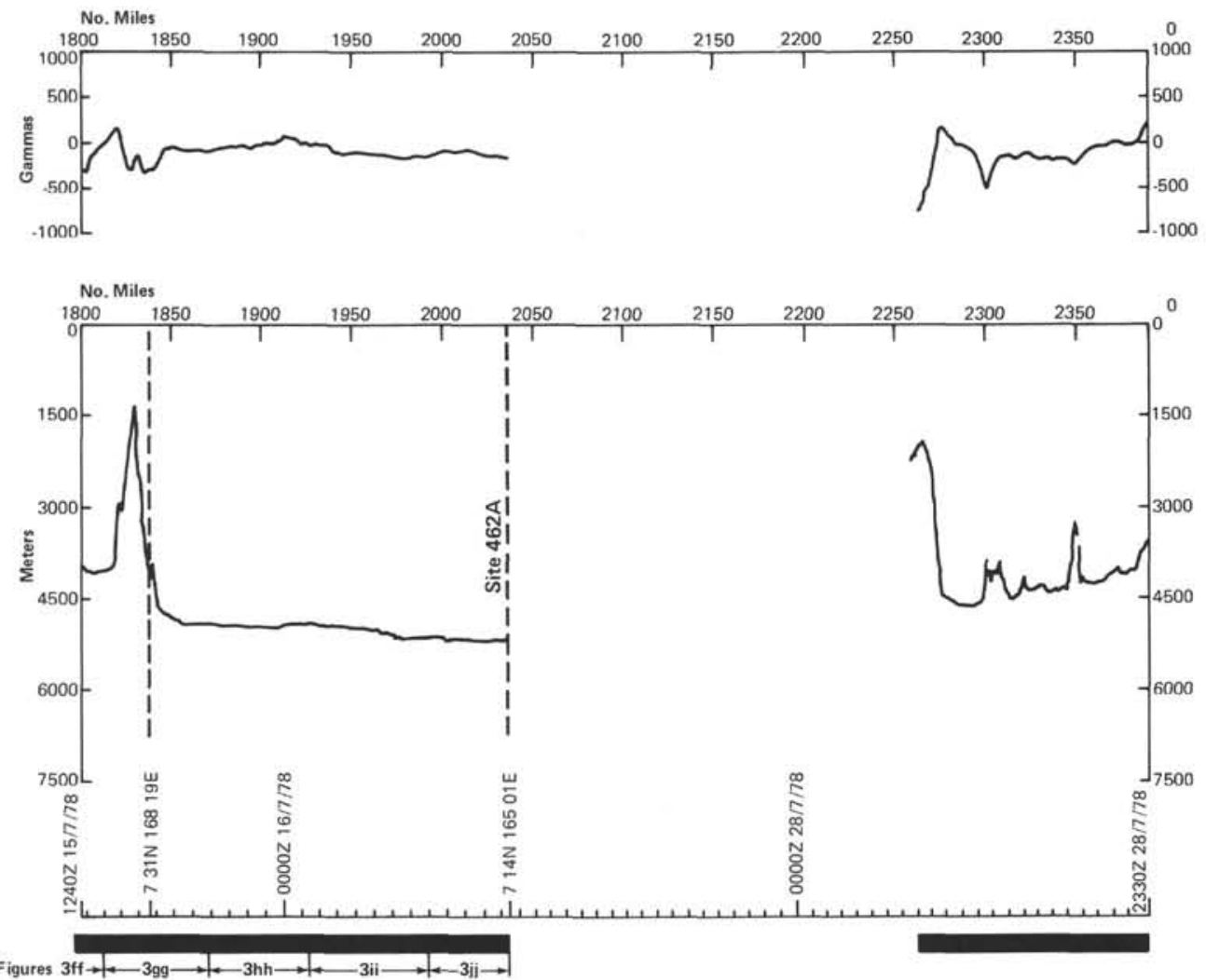

Figure 2. (Continued). 


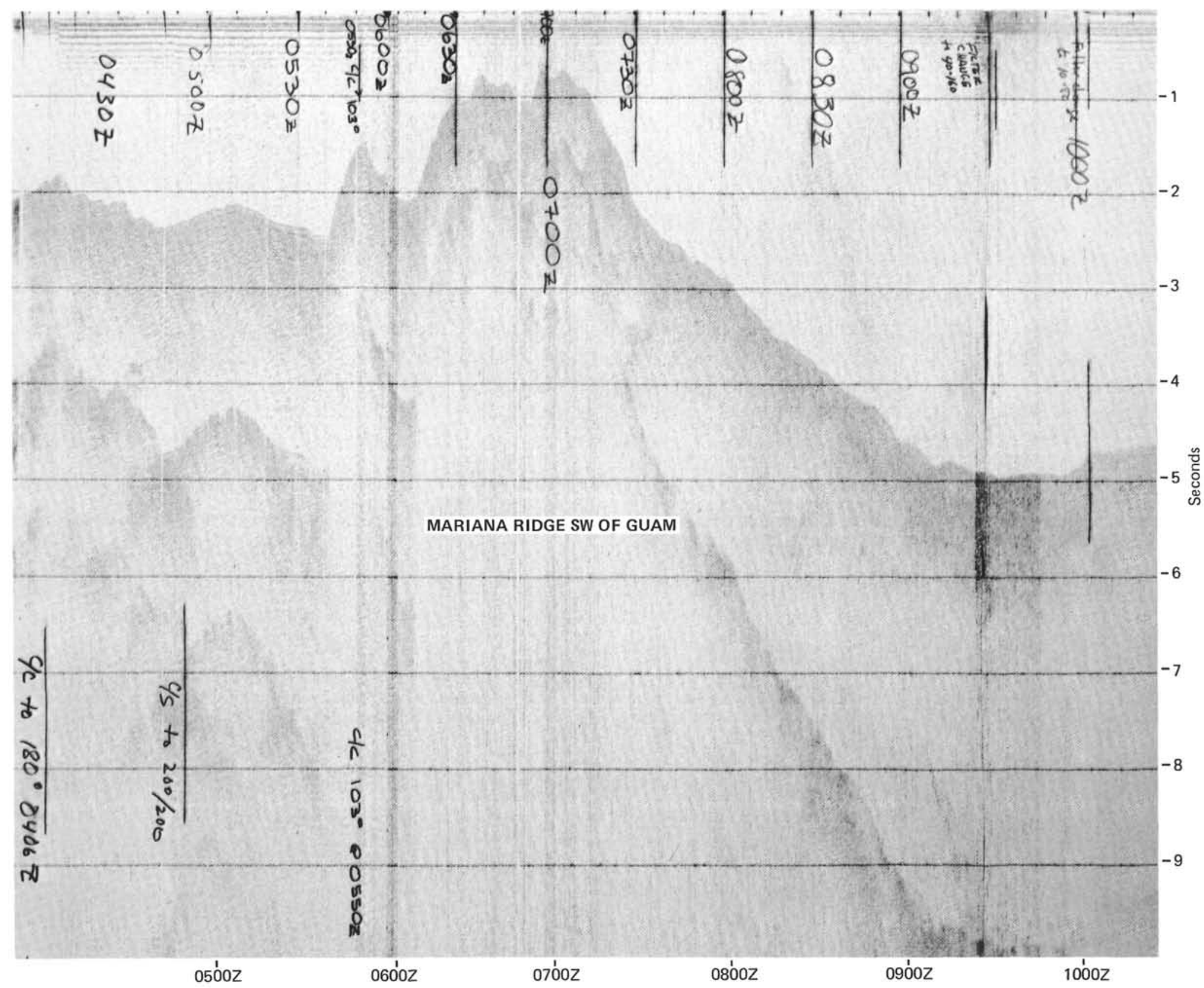




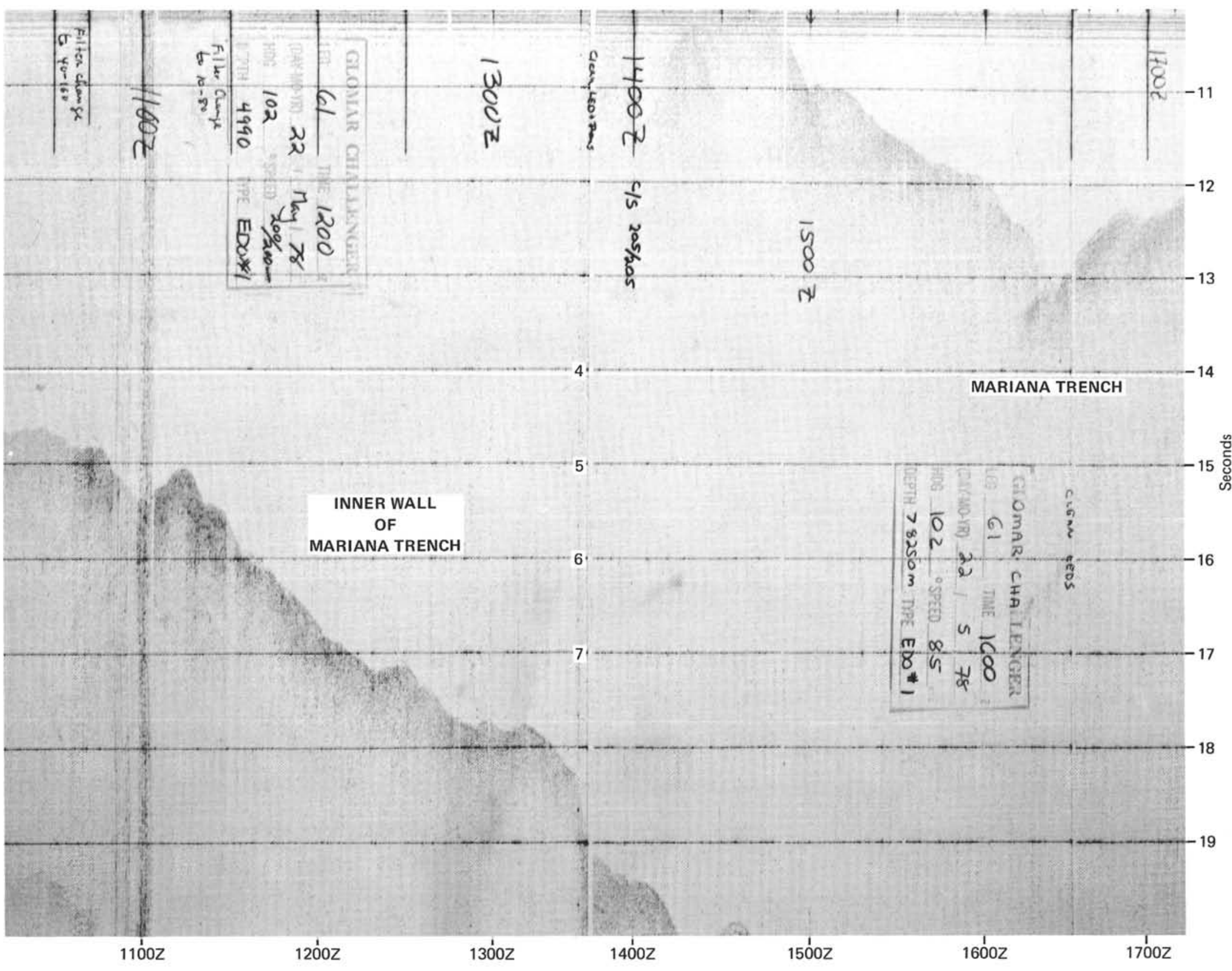




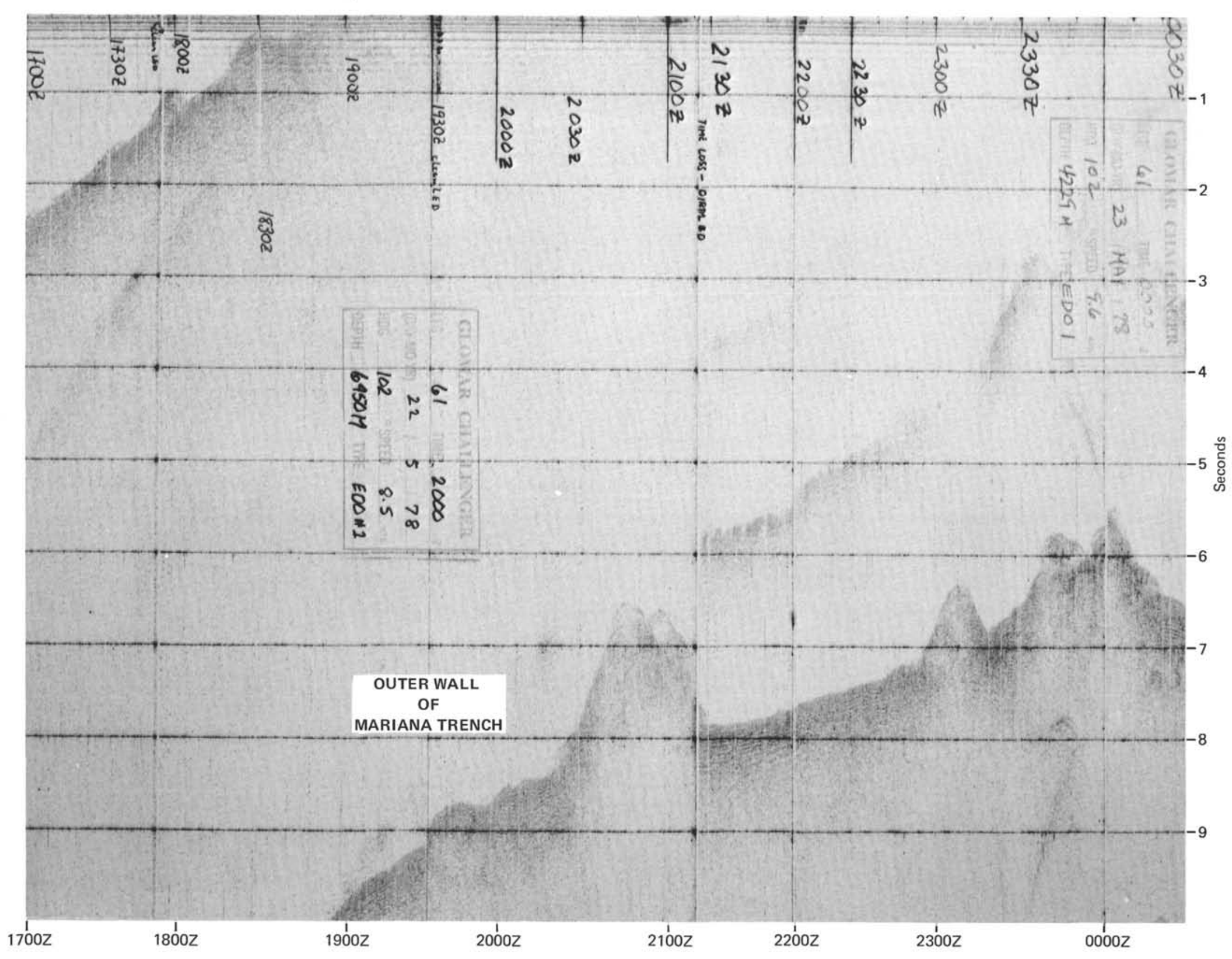




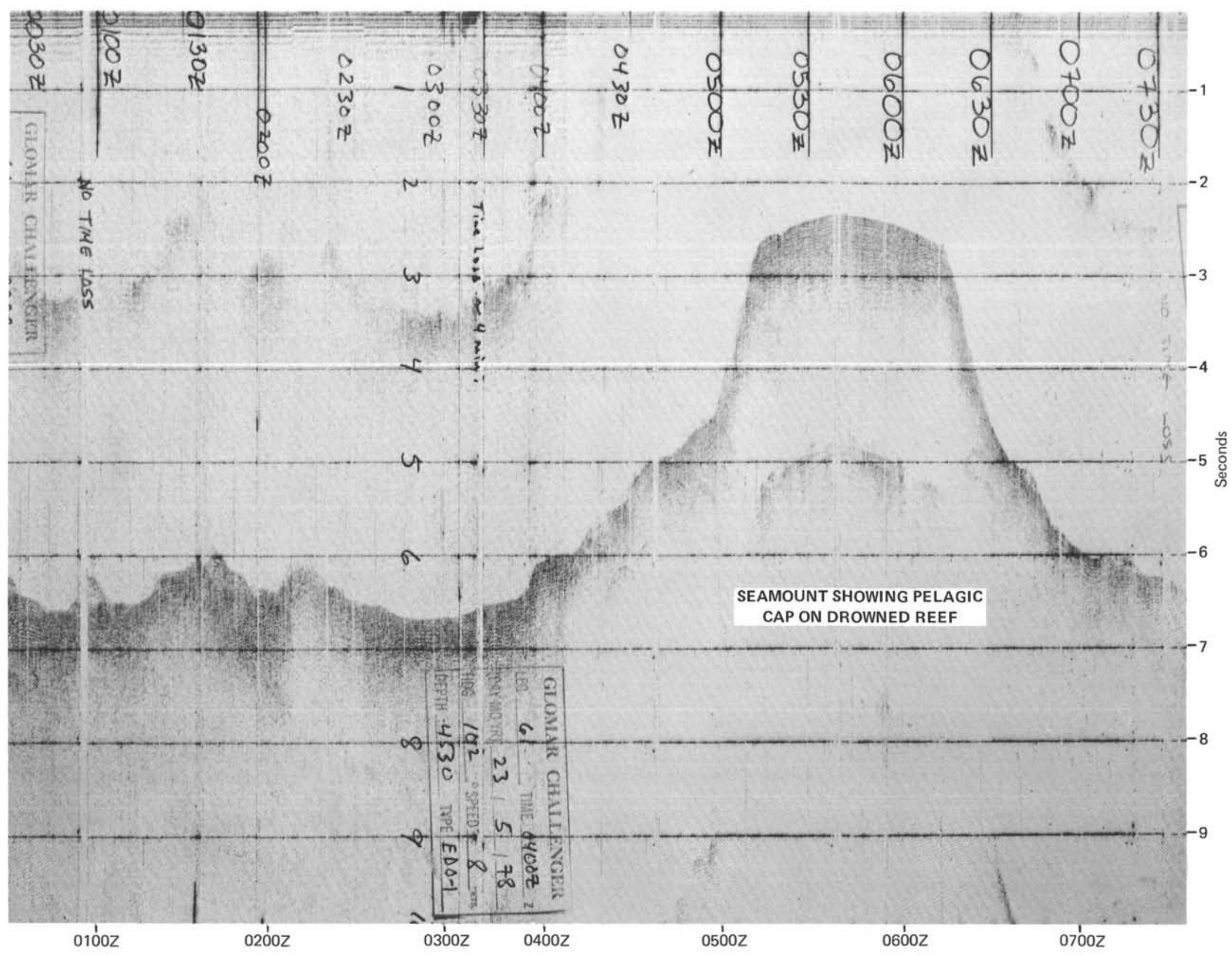



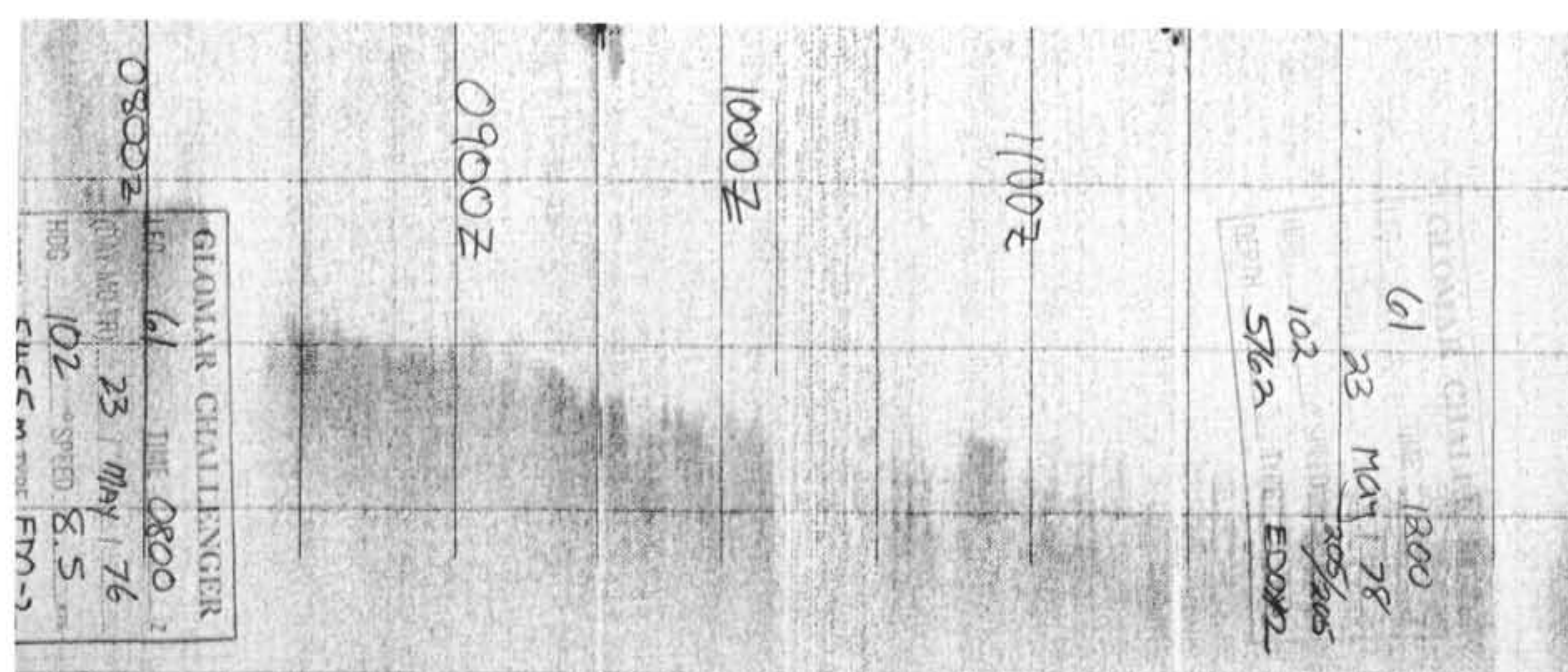

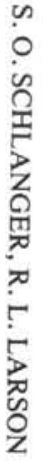

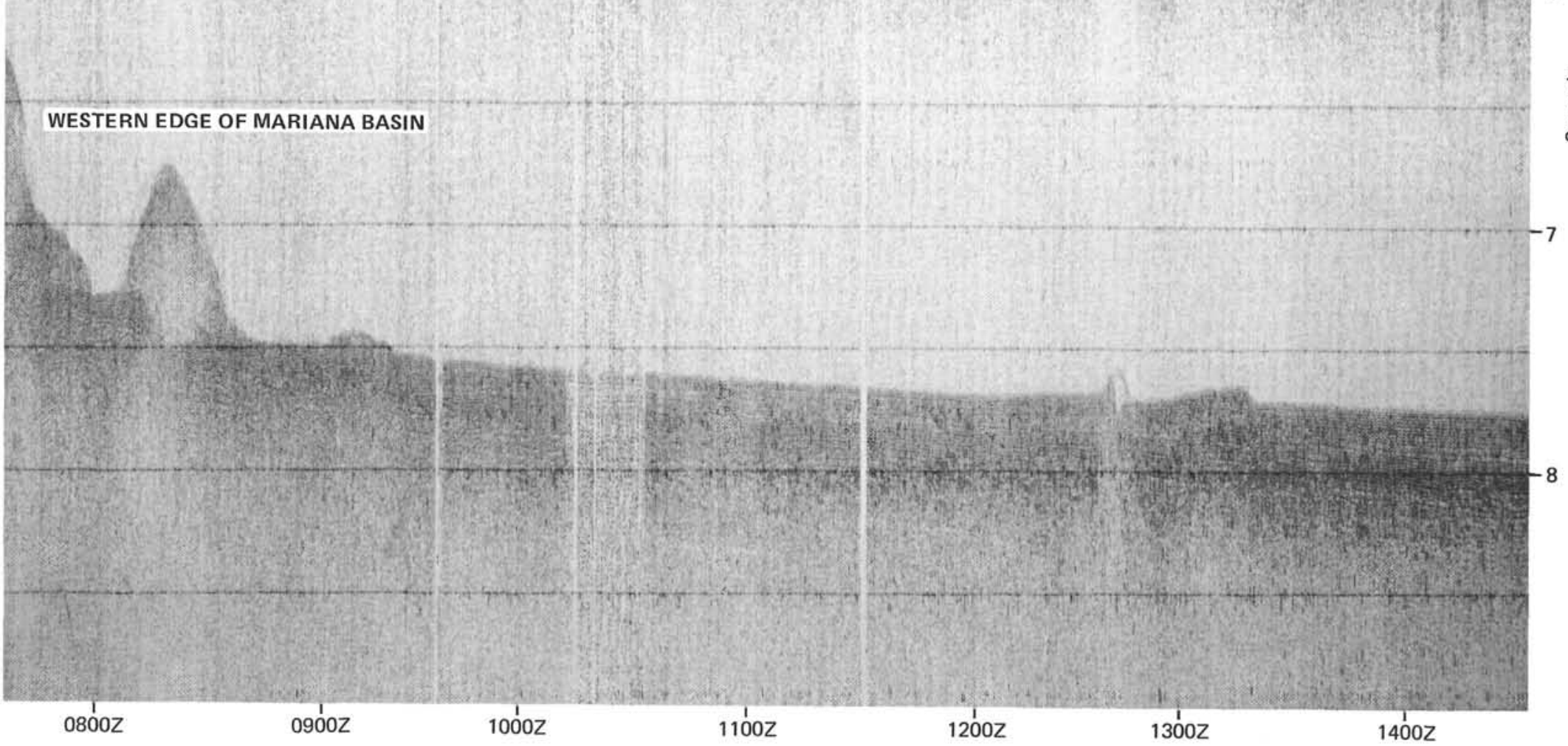



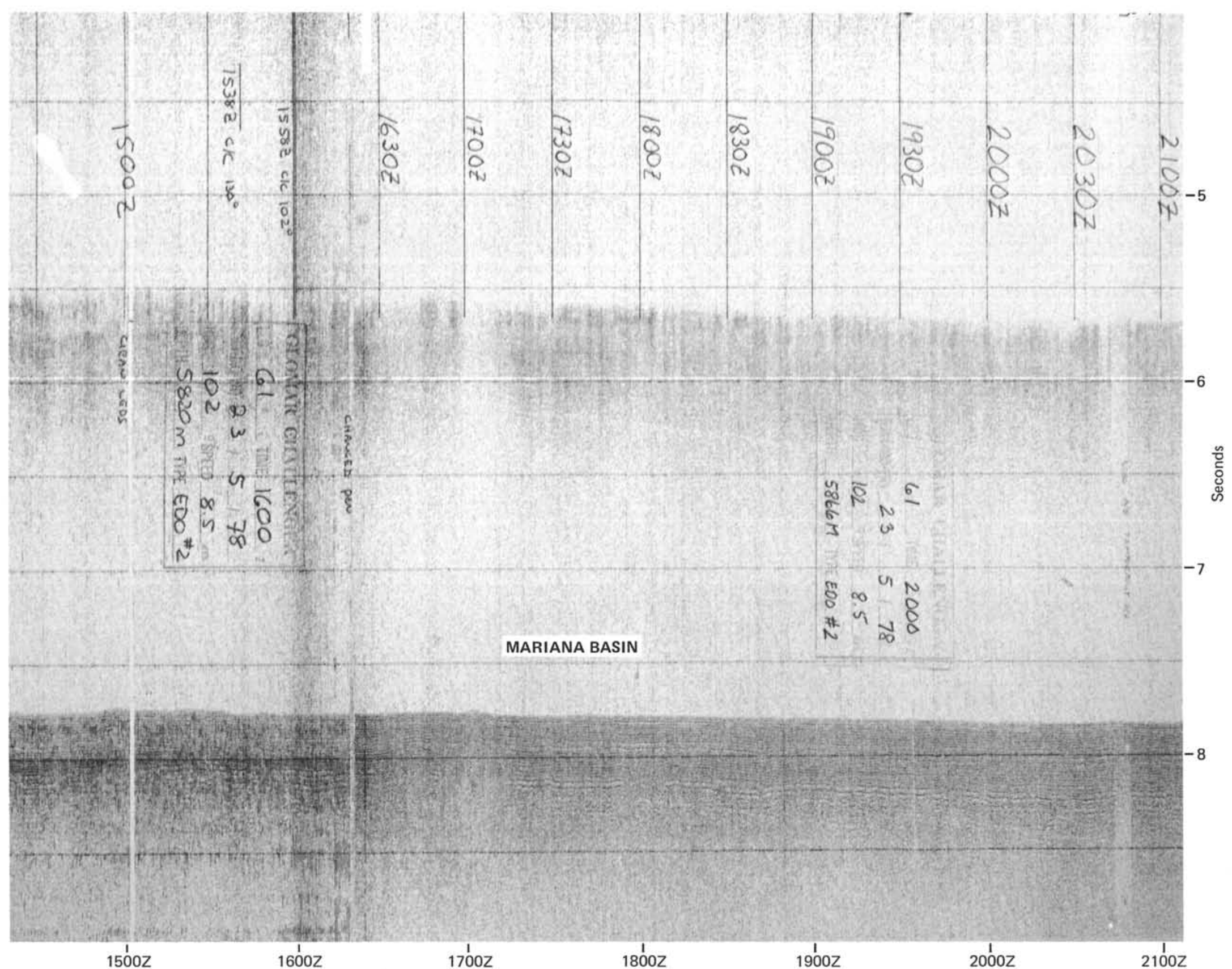

Figure 3. (Continued). 


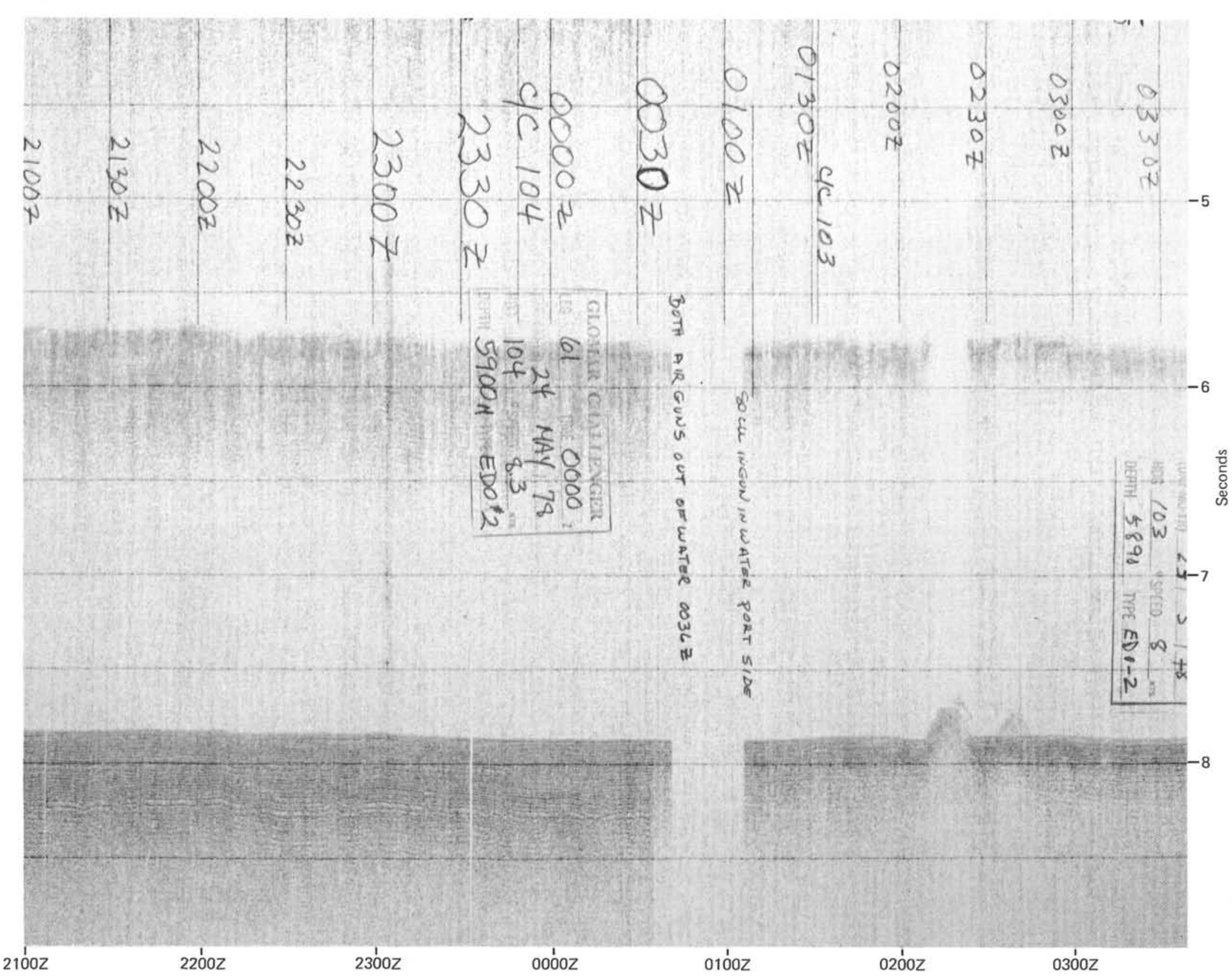




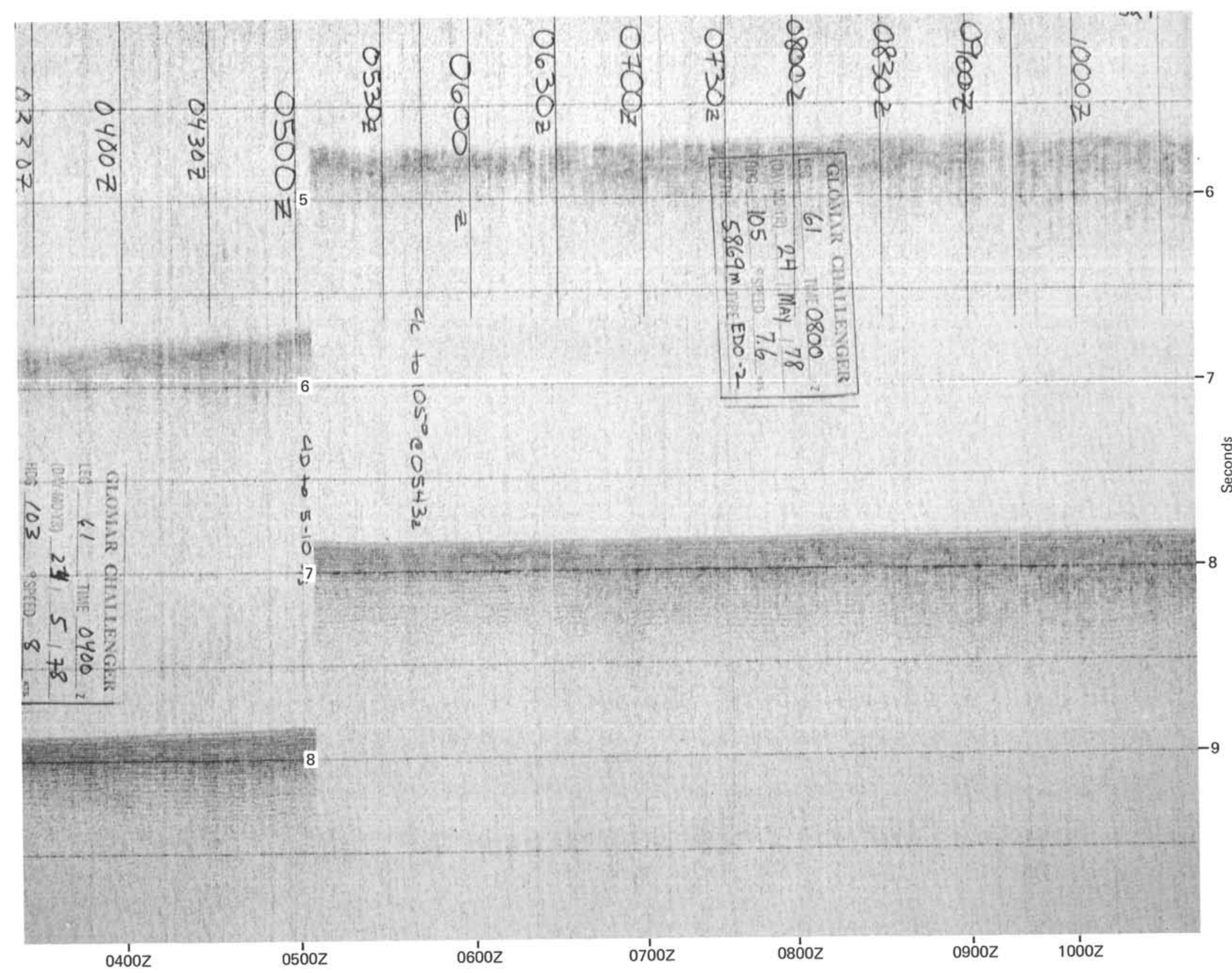




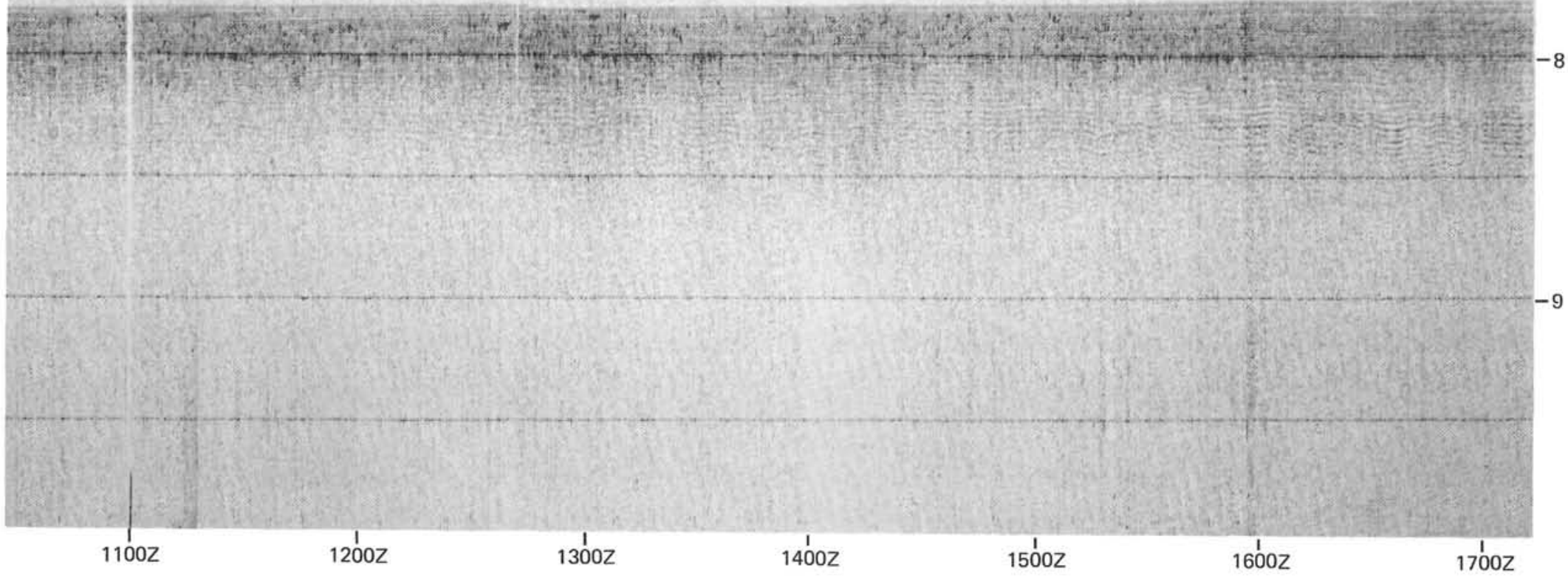



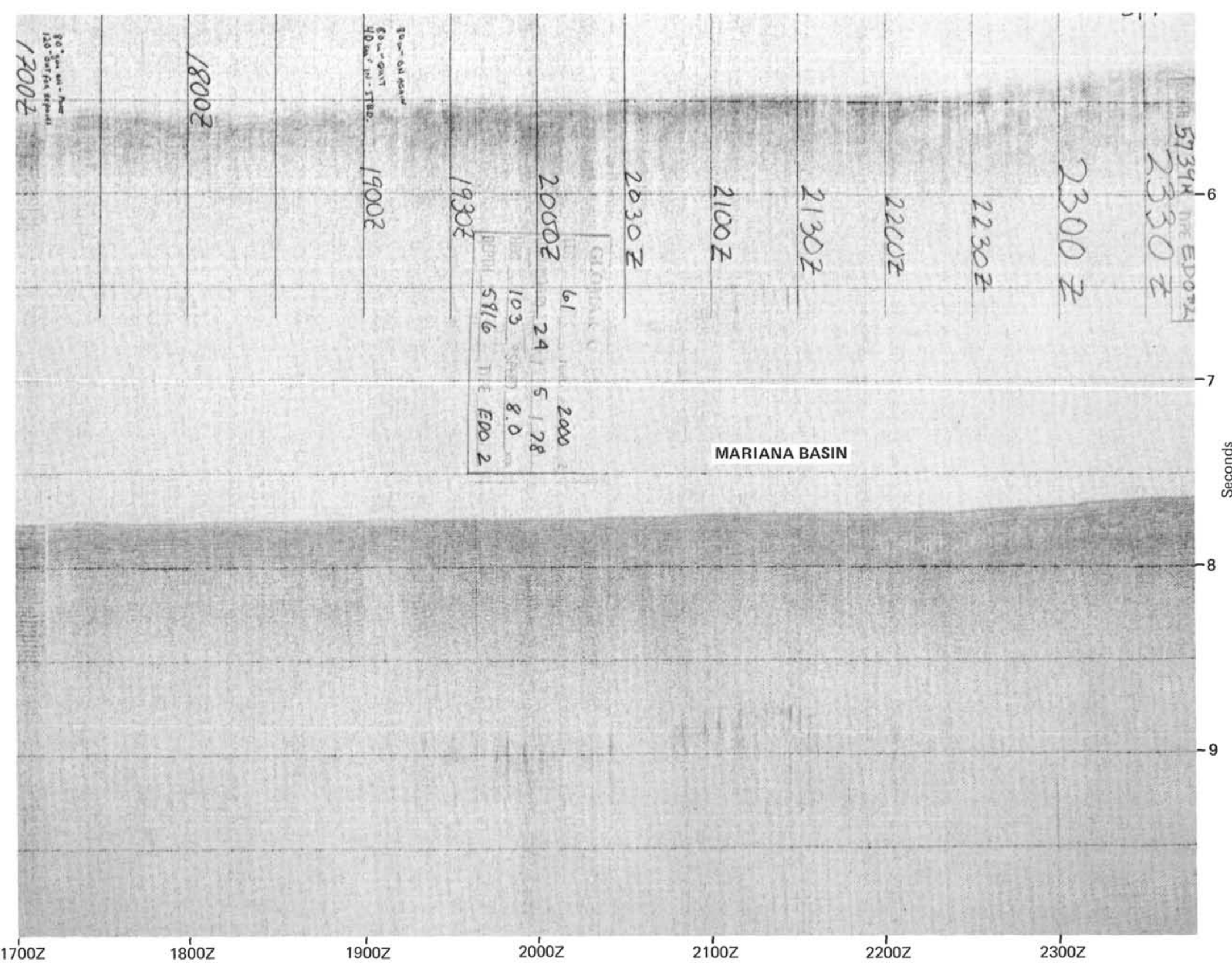


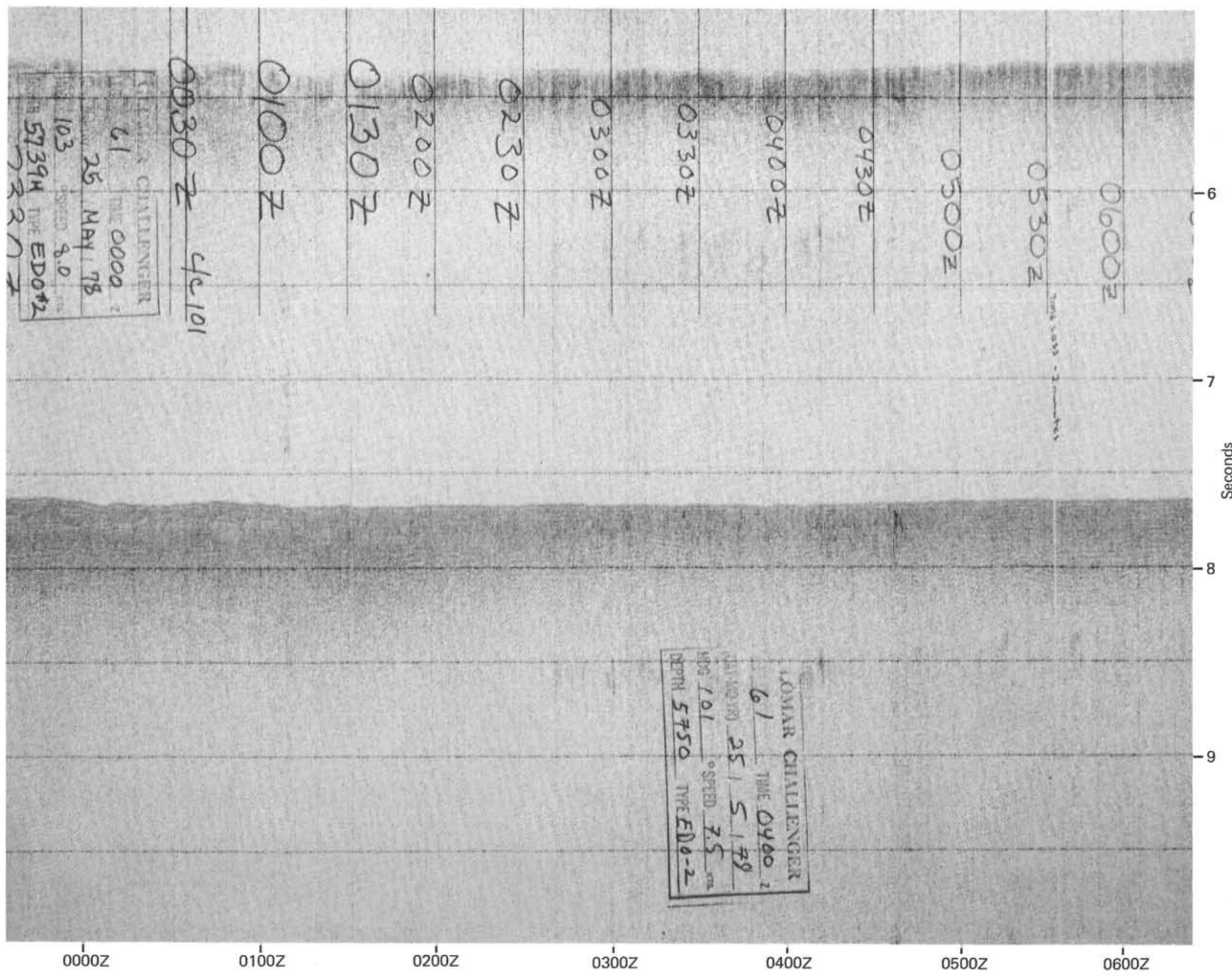




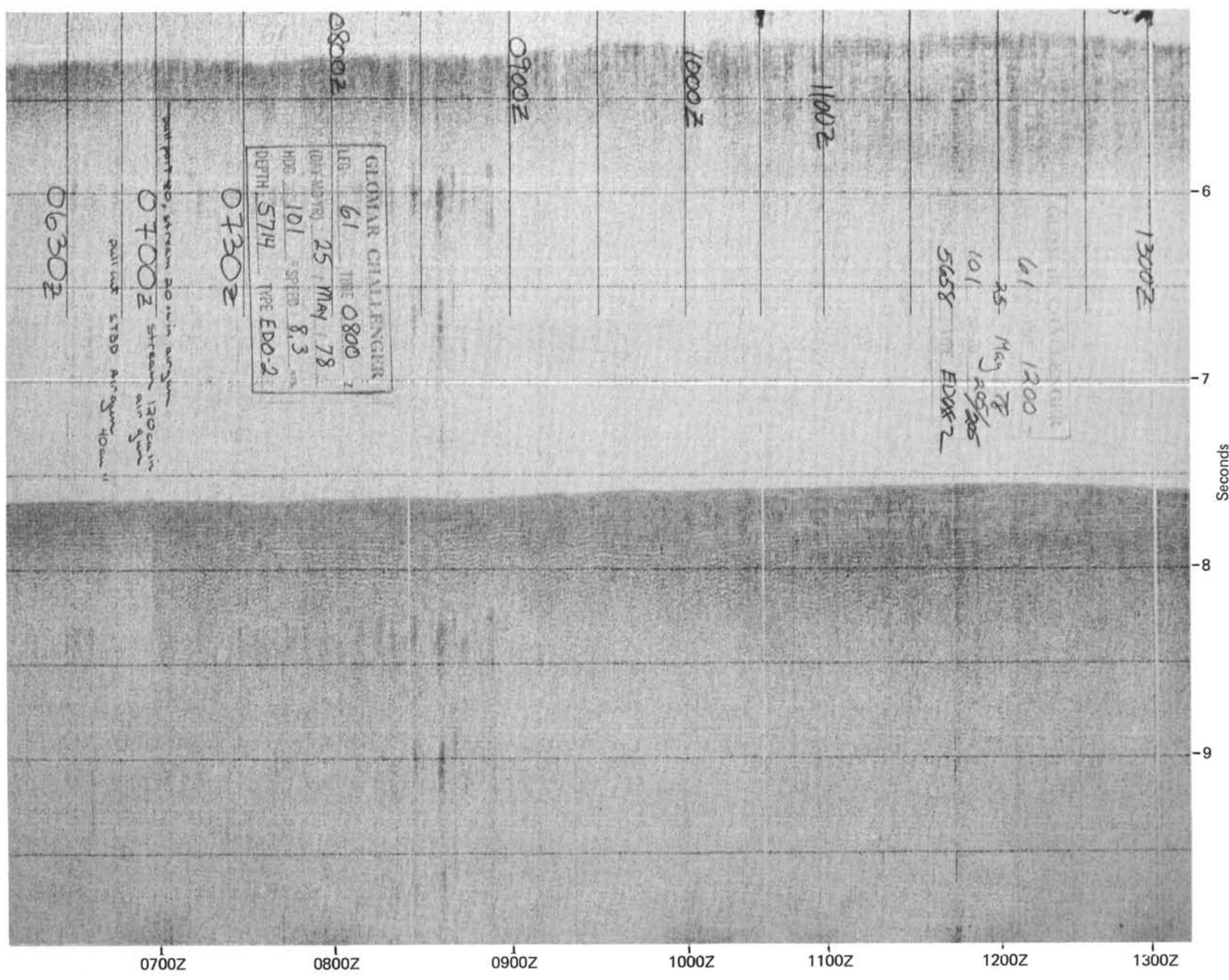




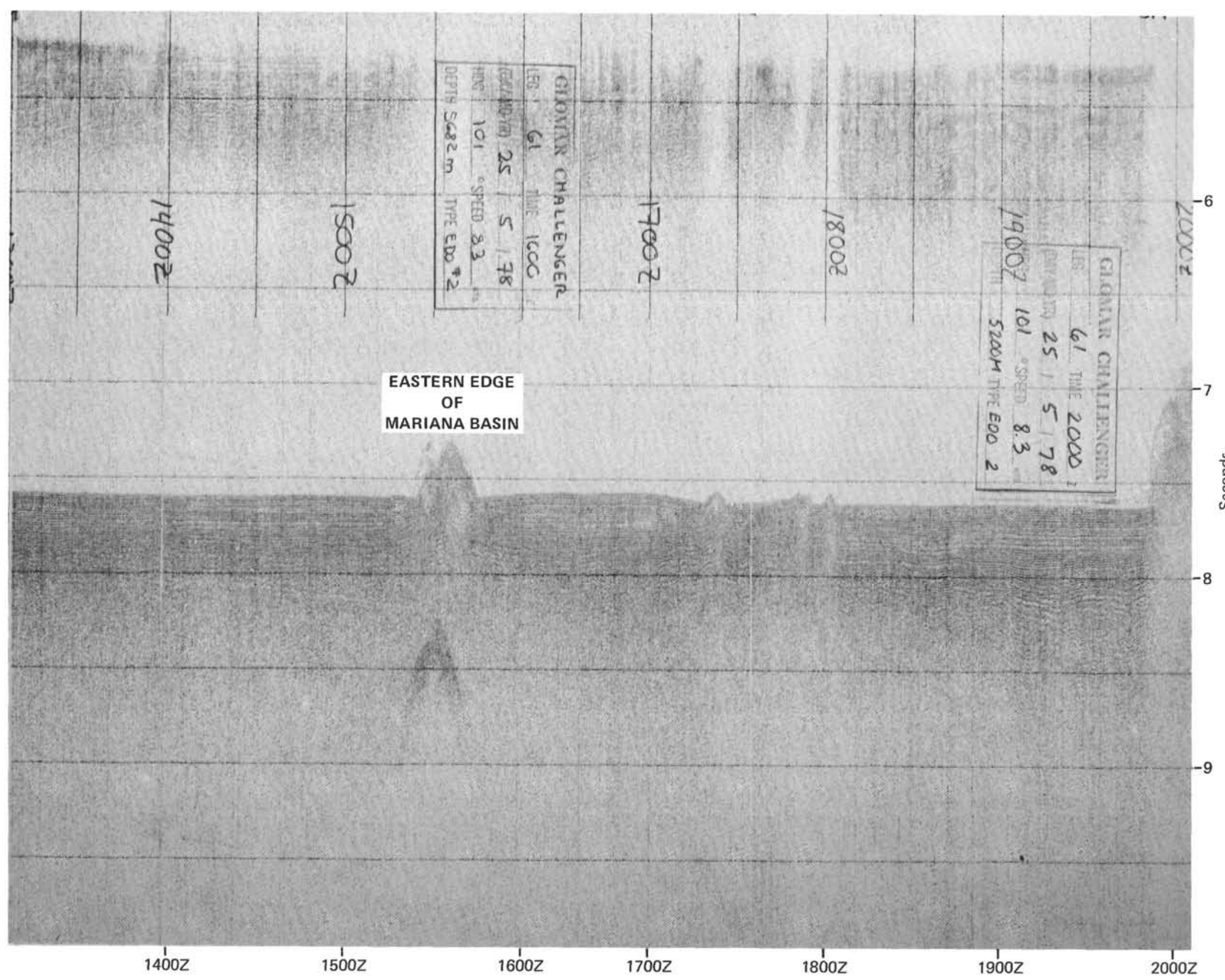




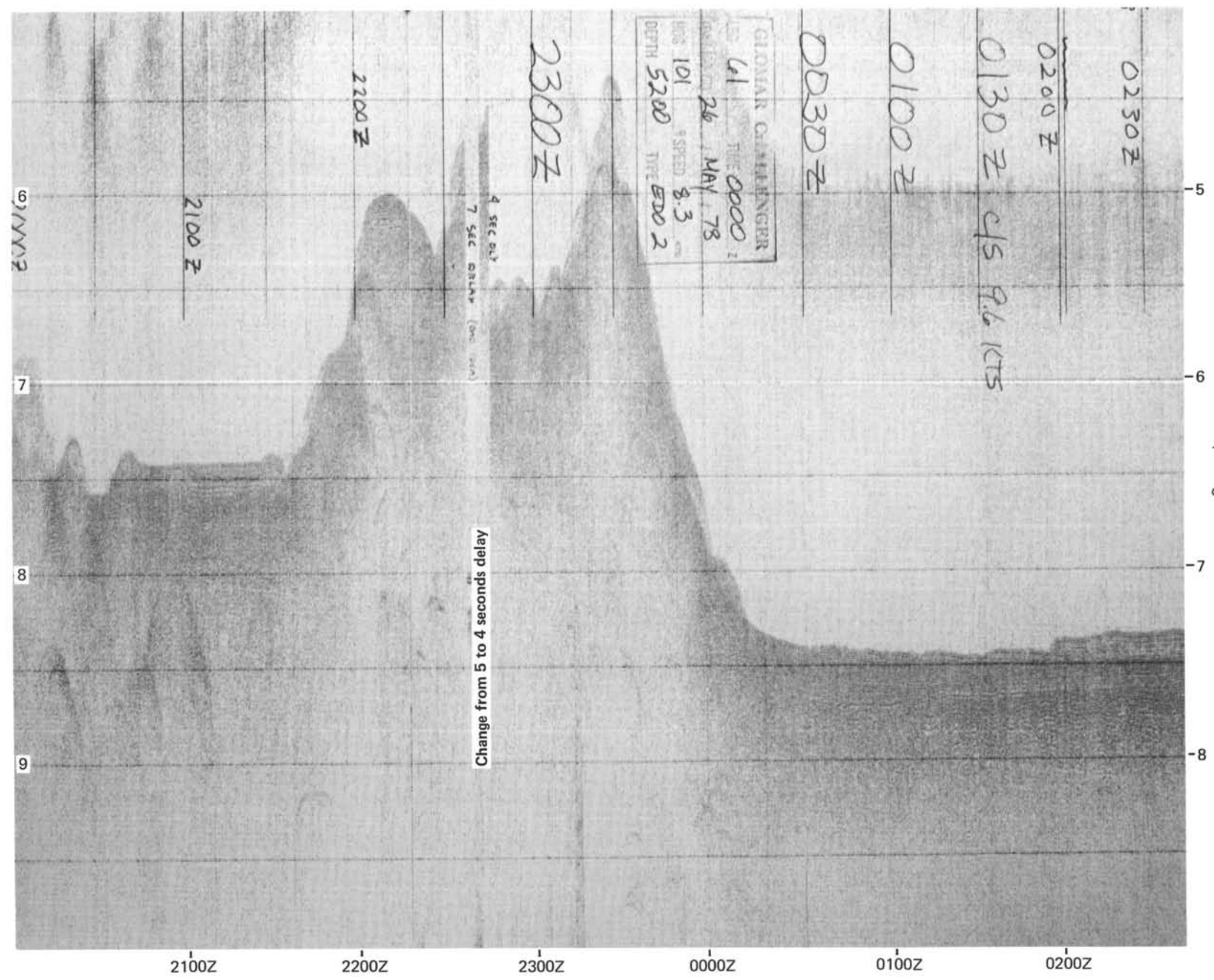




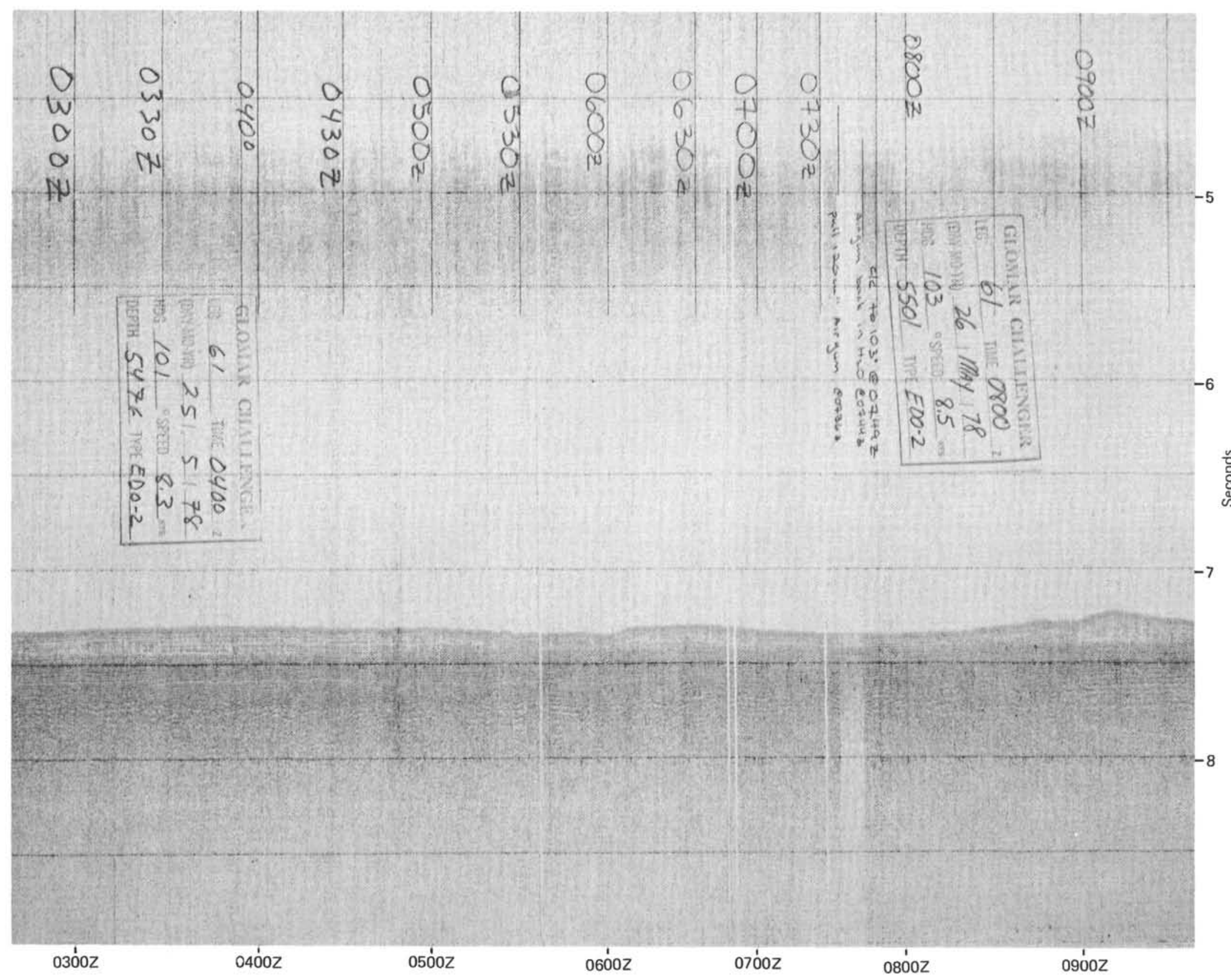




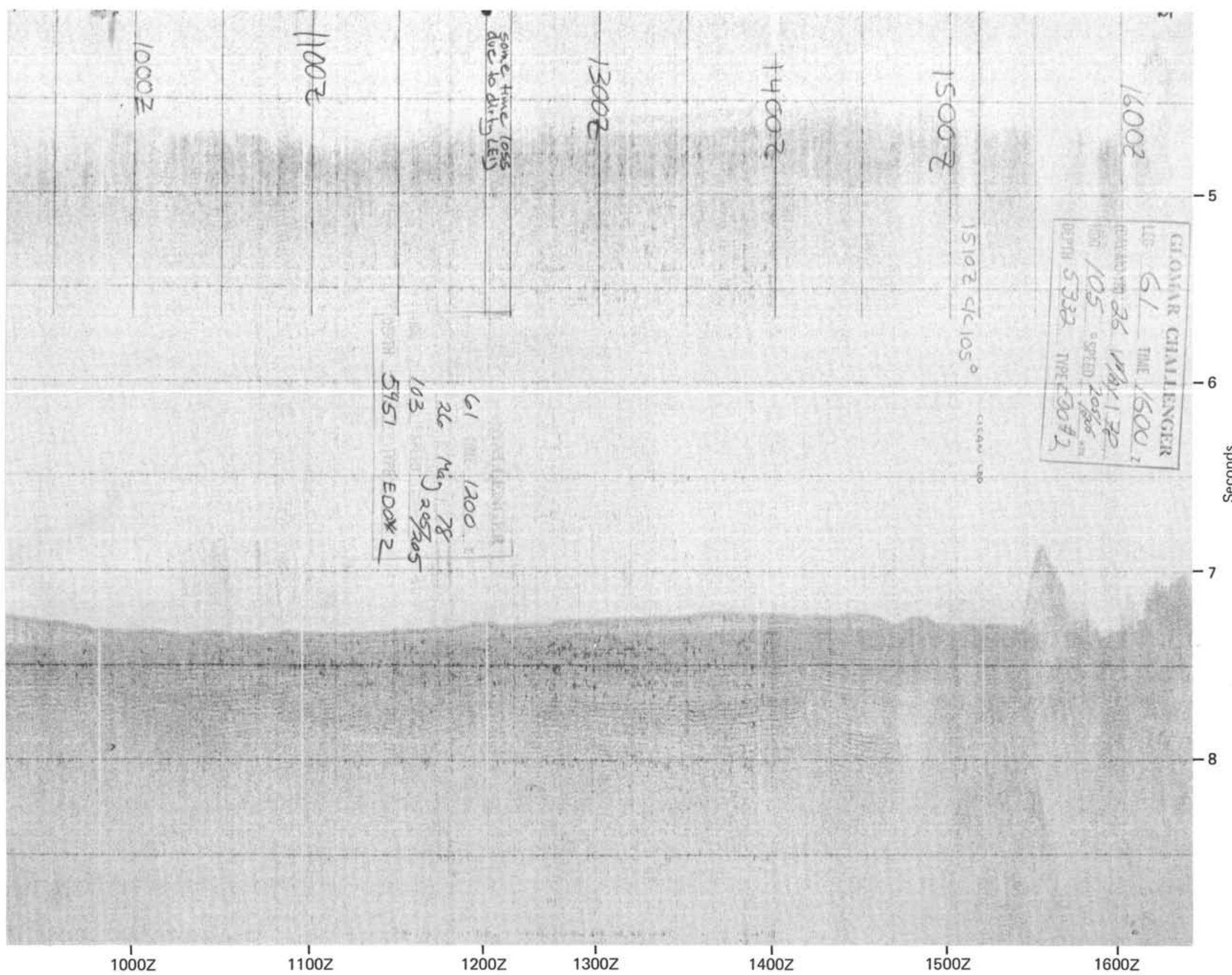




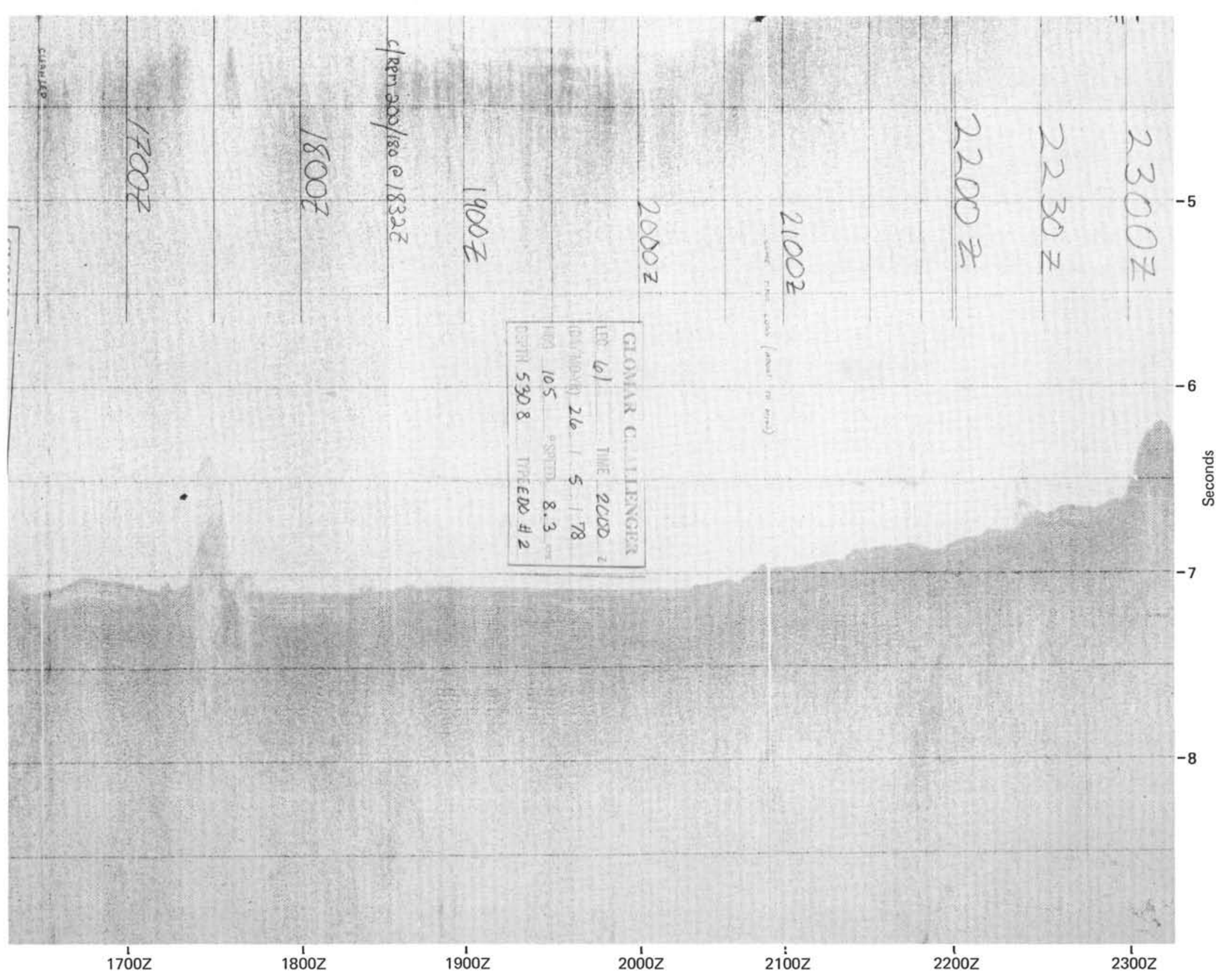




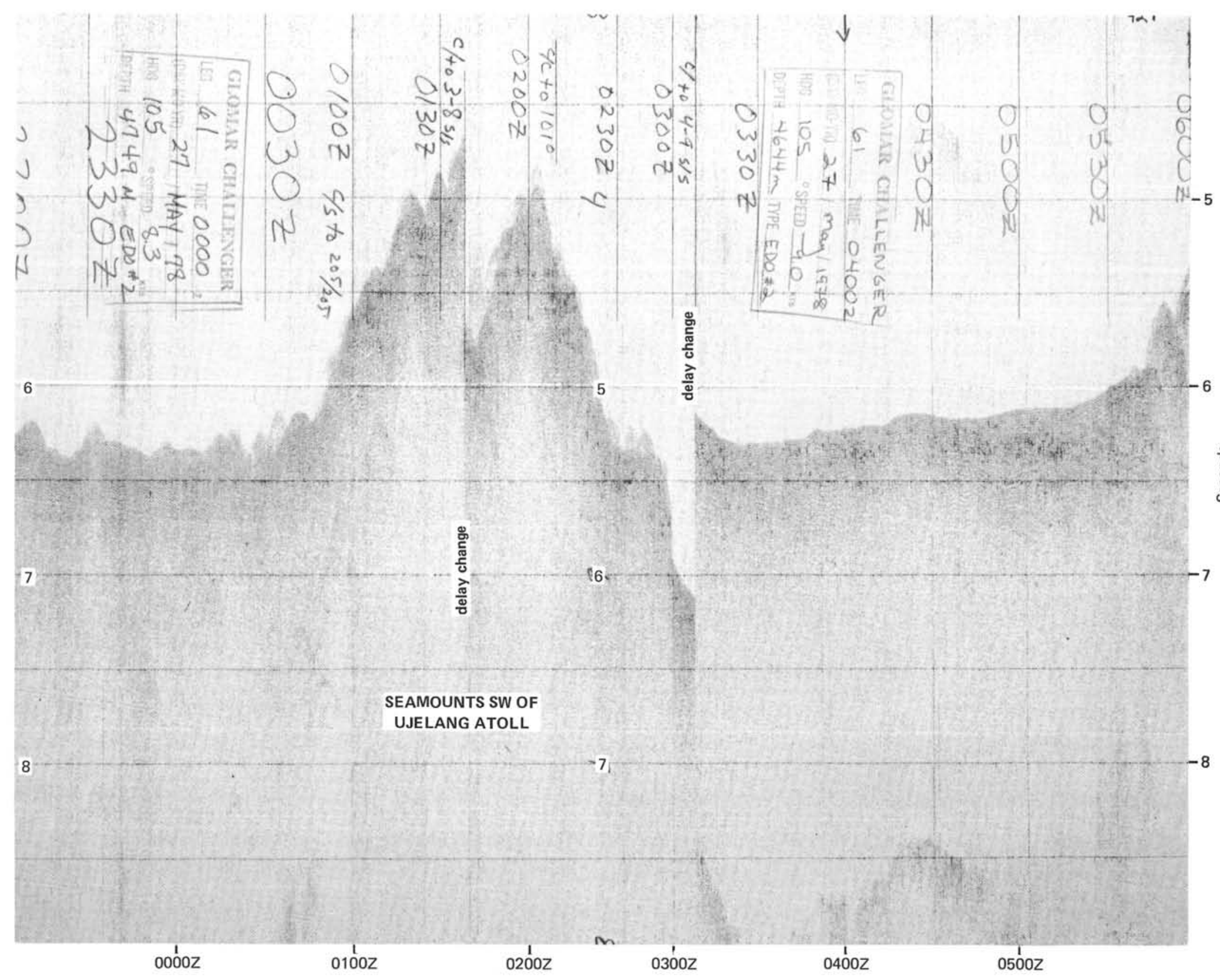




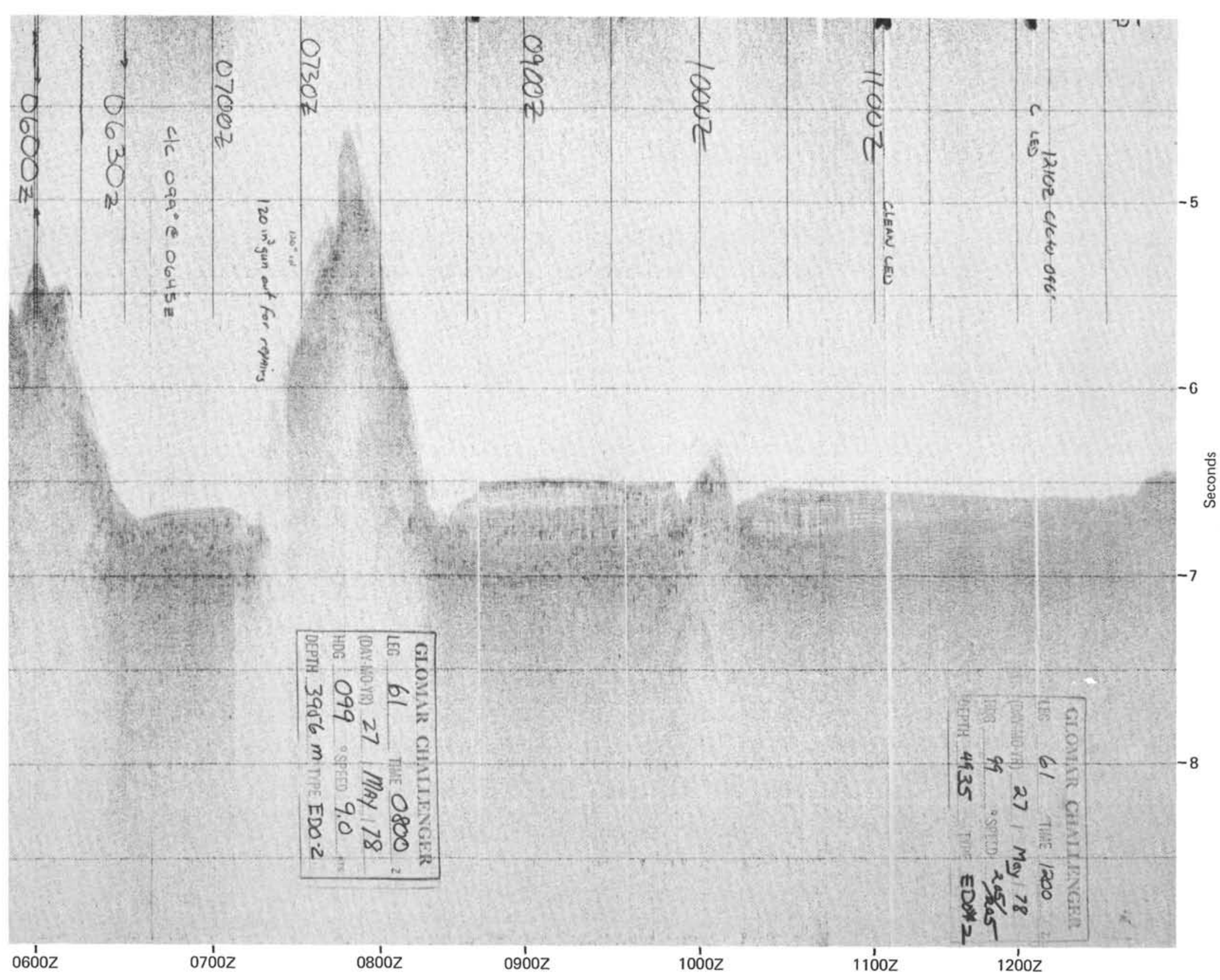




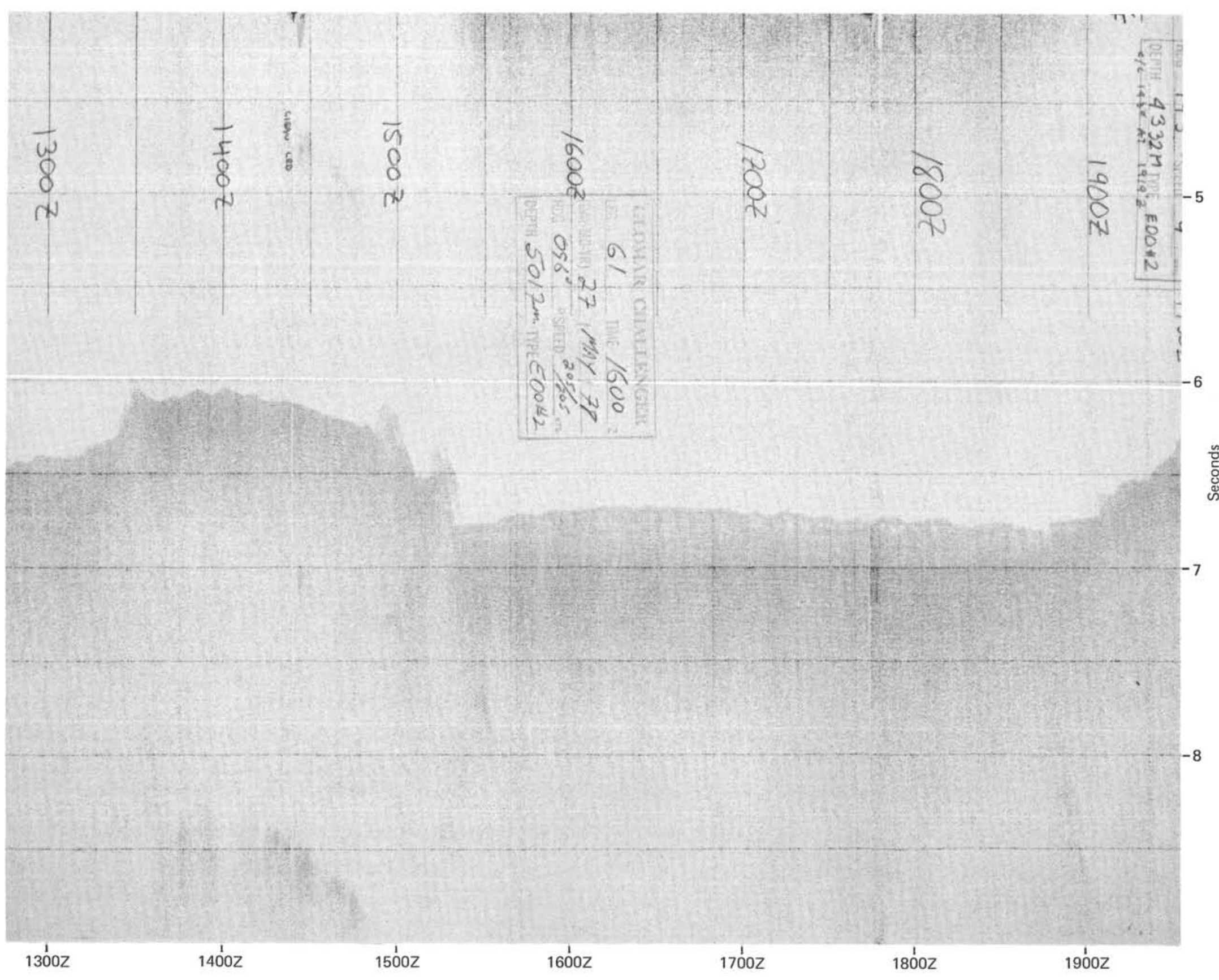




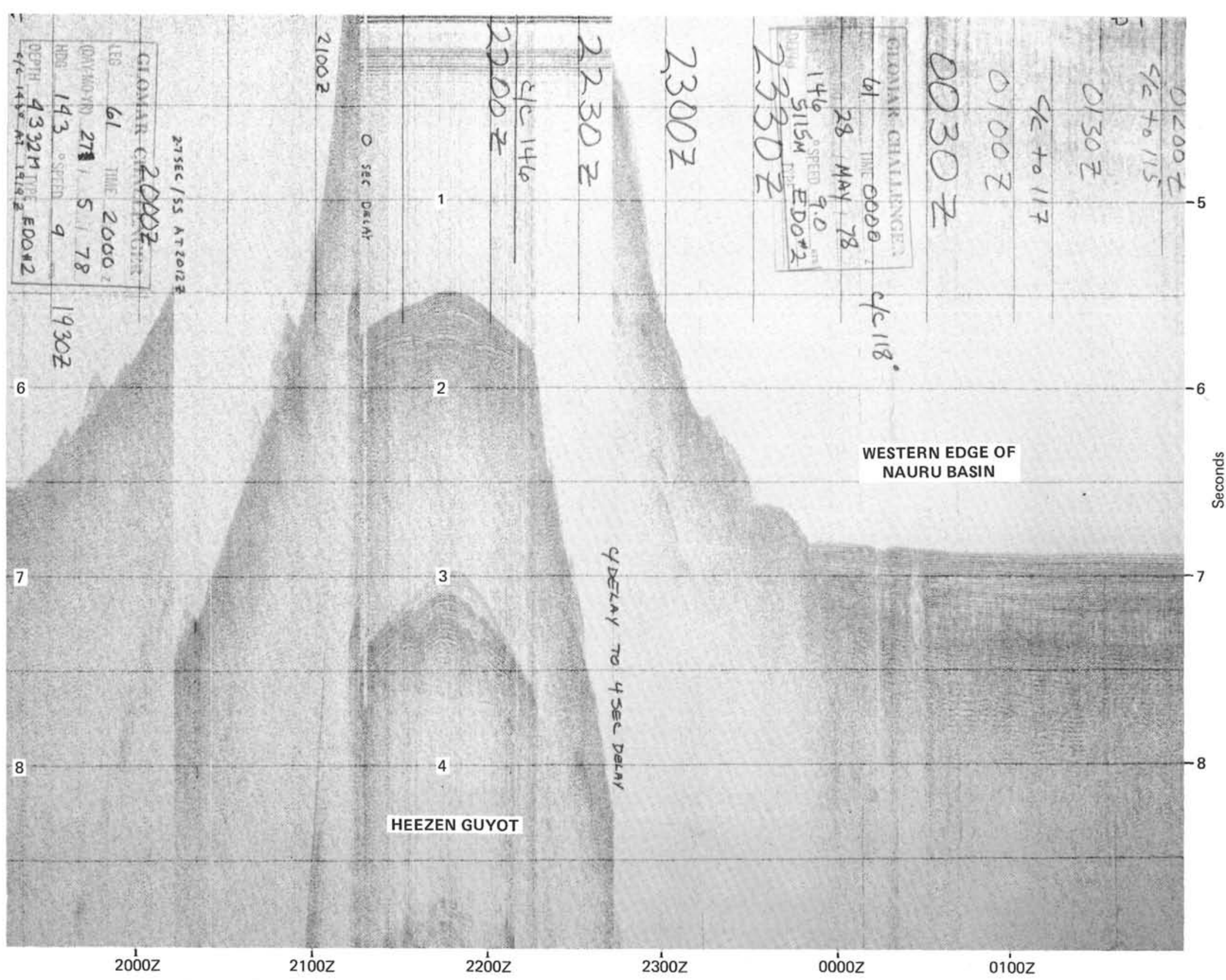




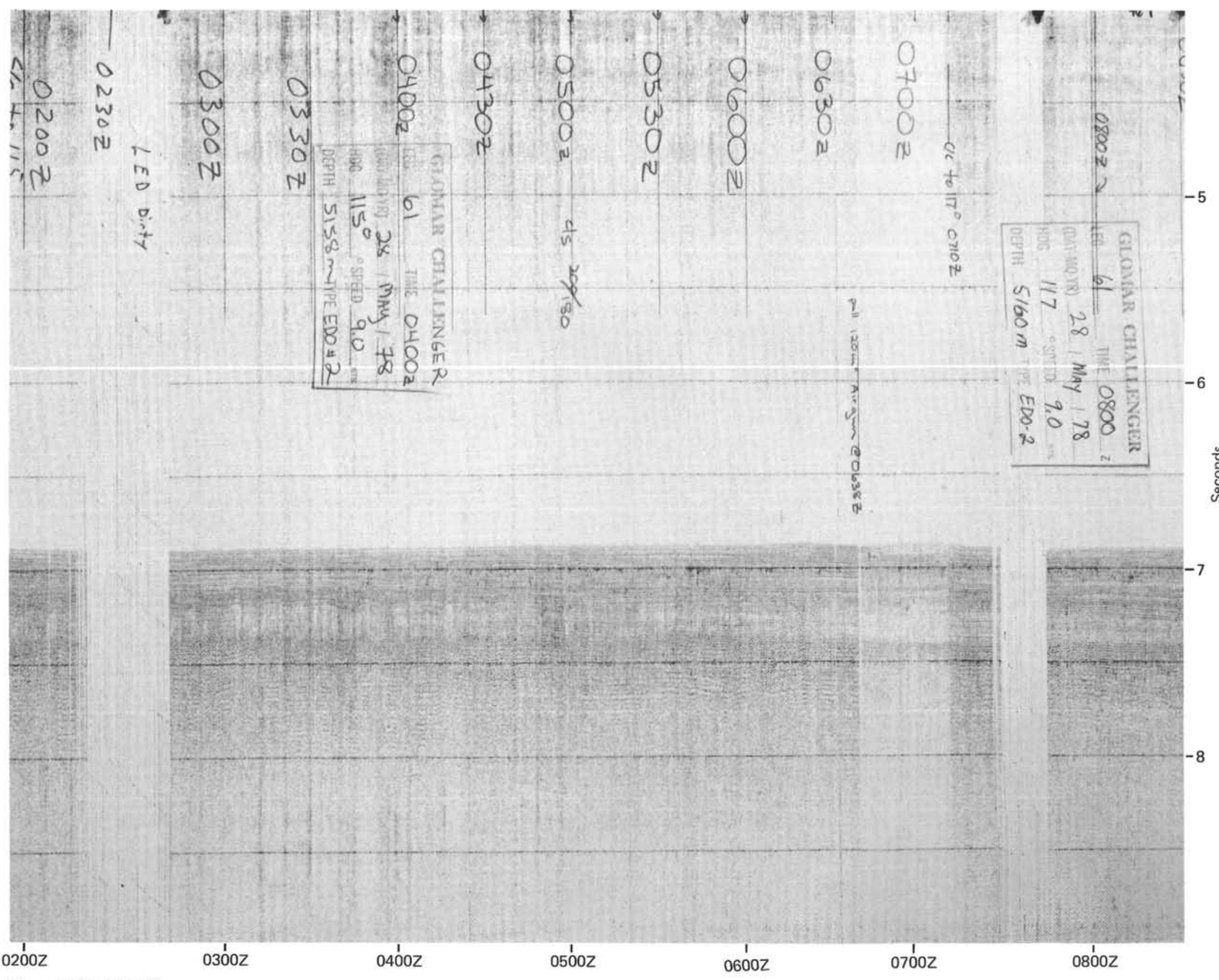



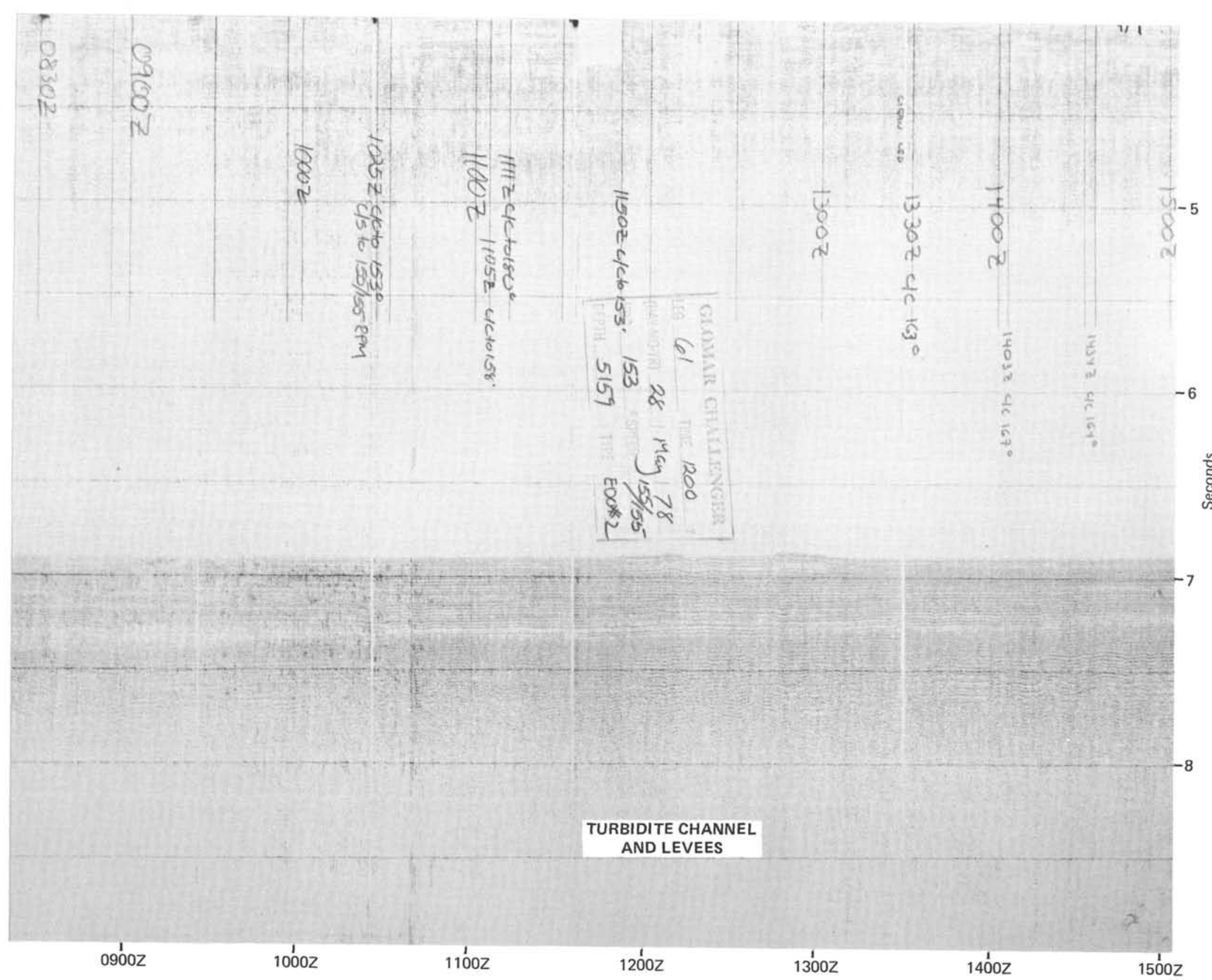


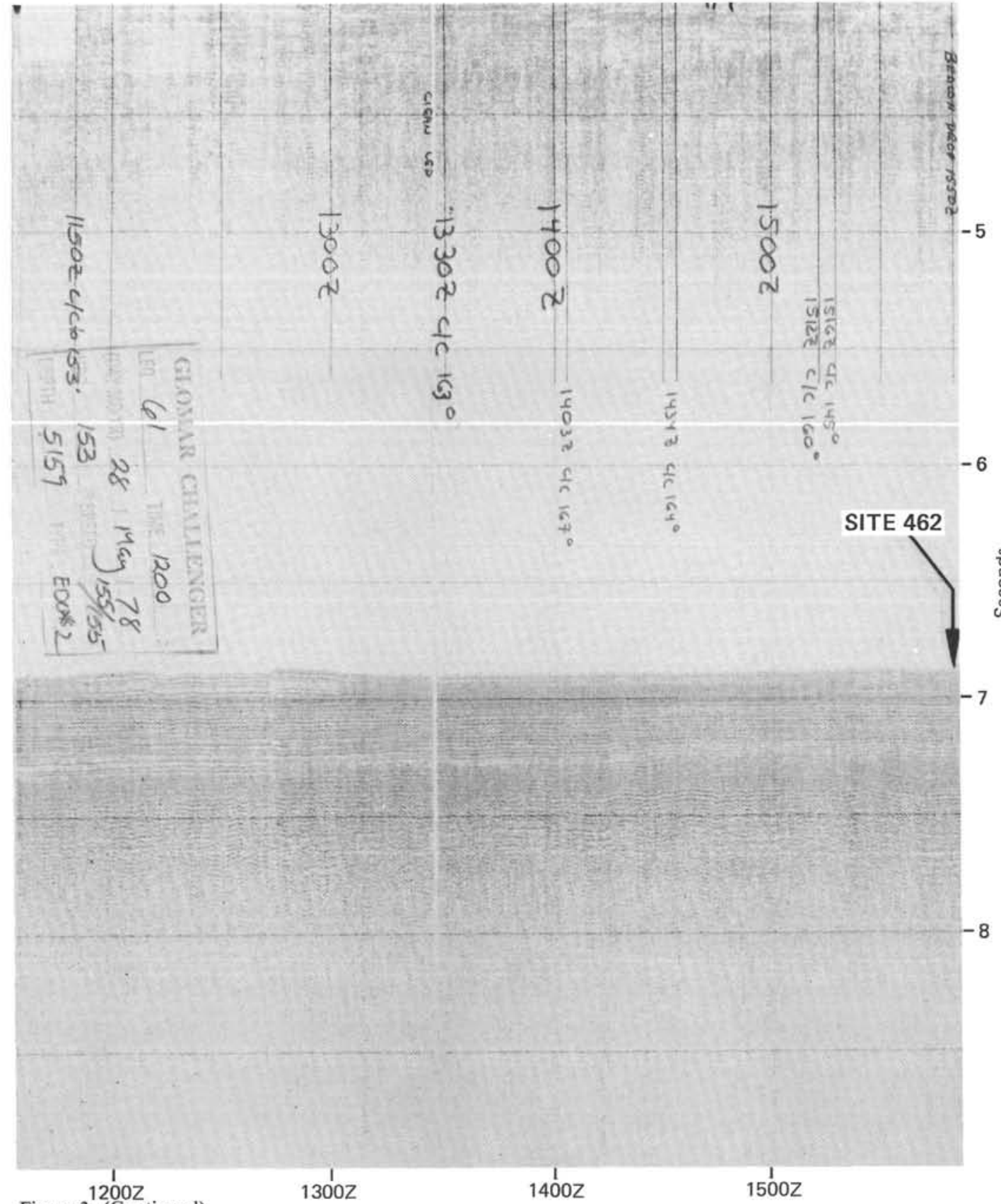




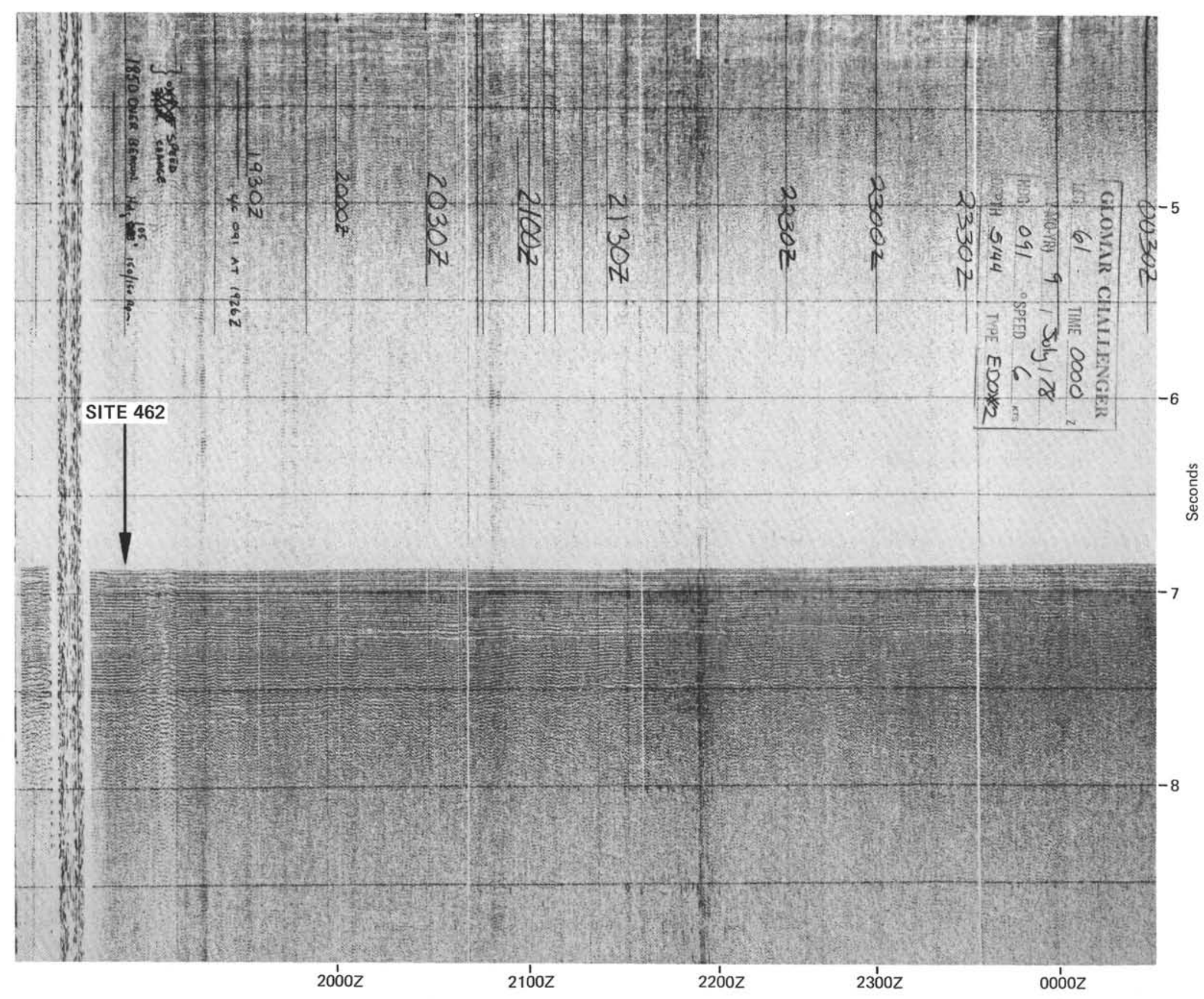




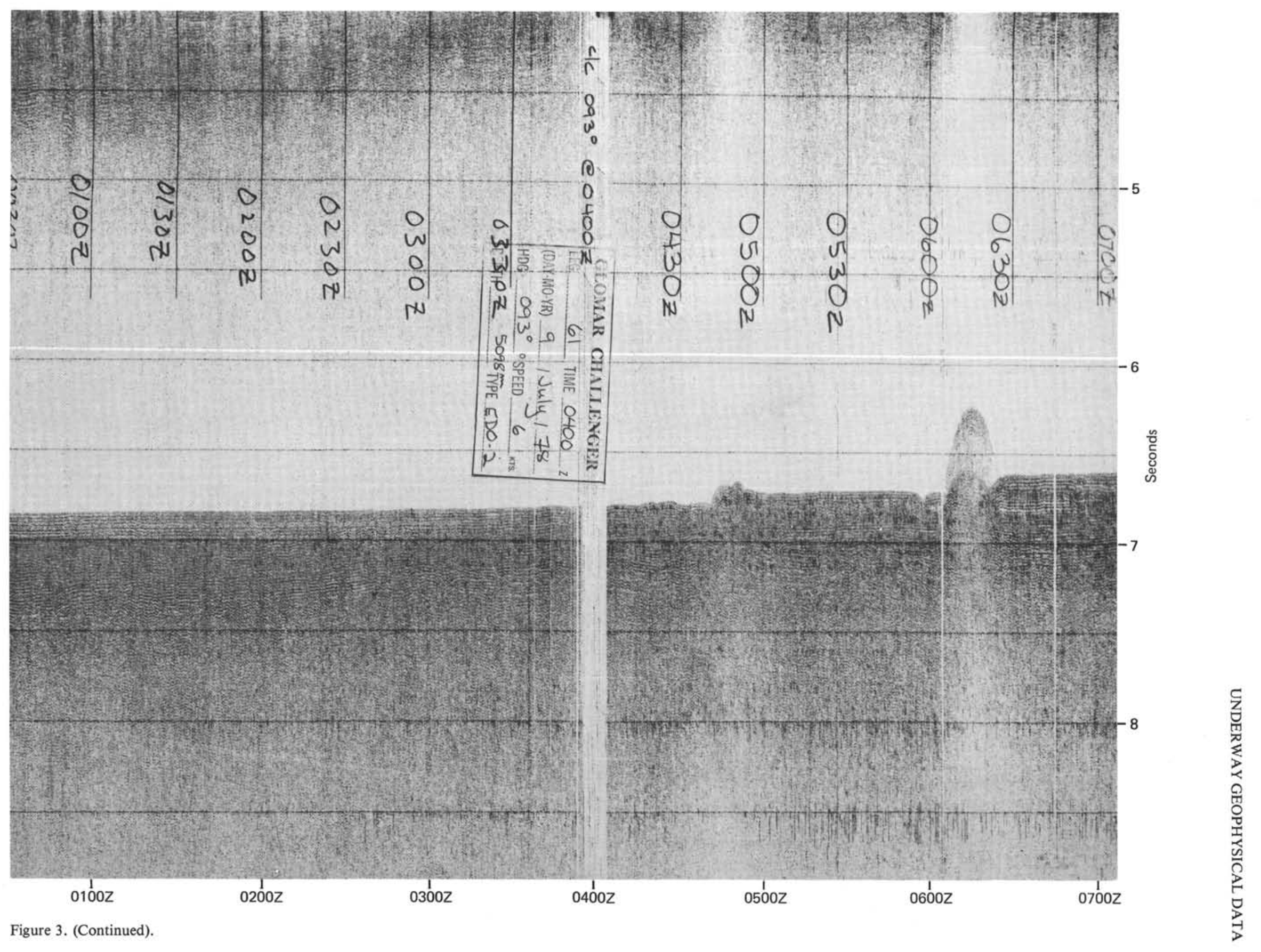




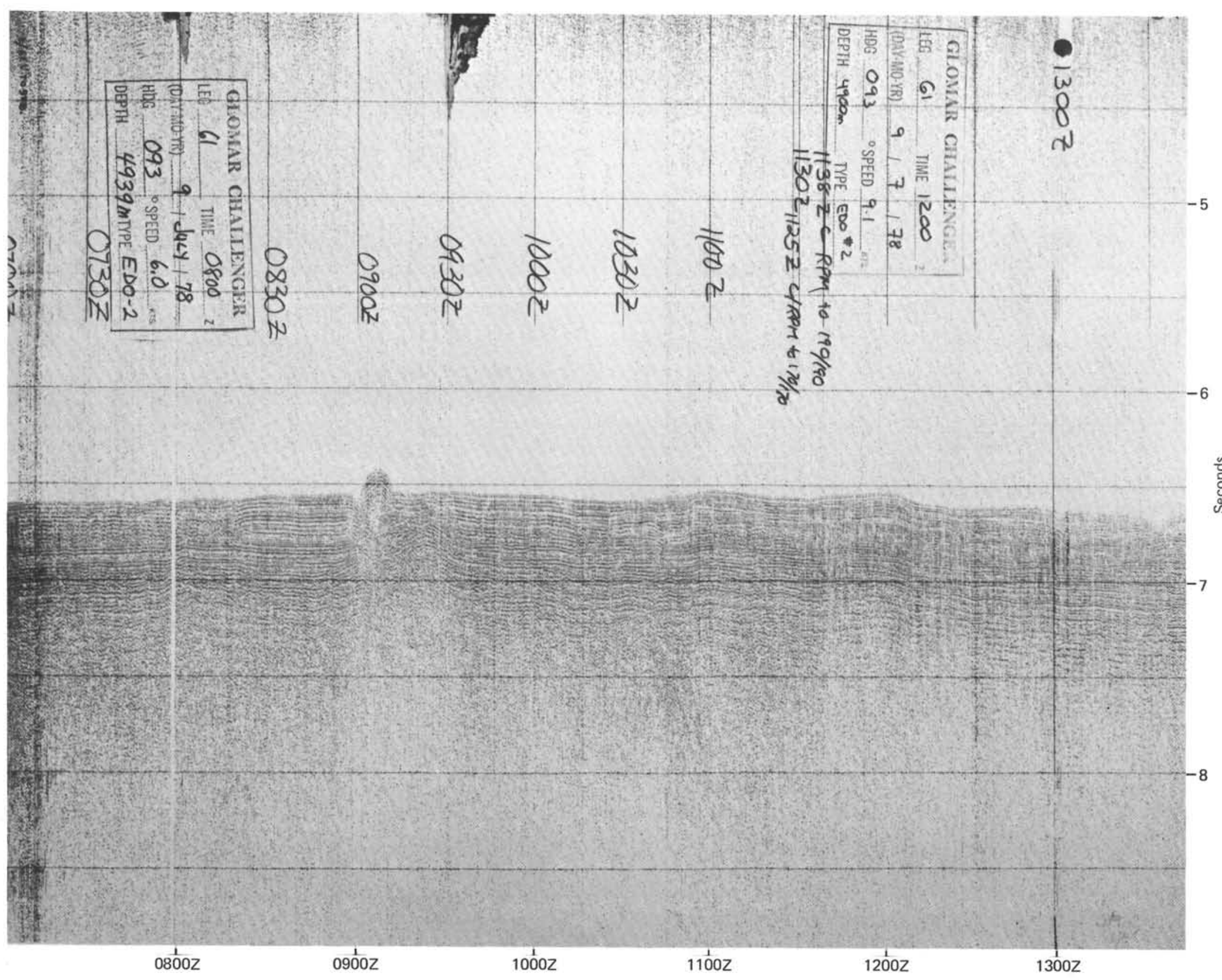




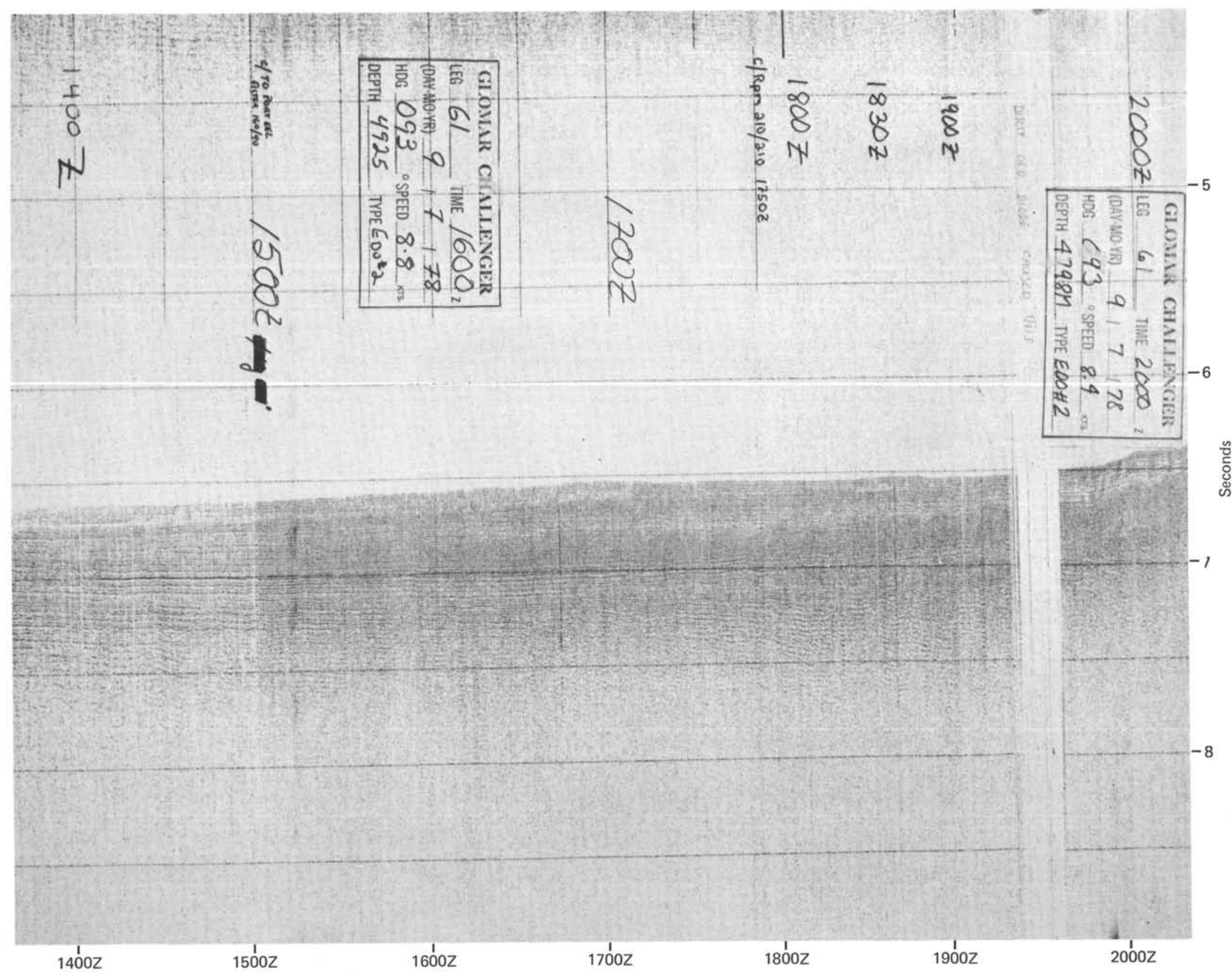




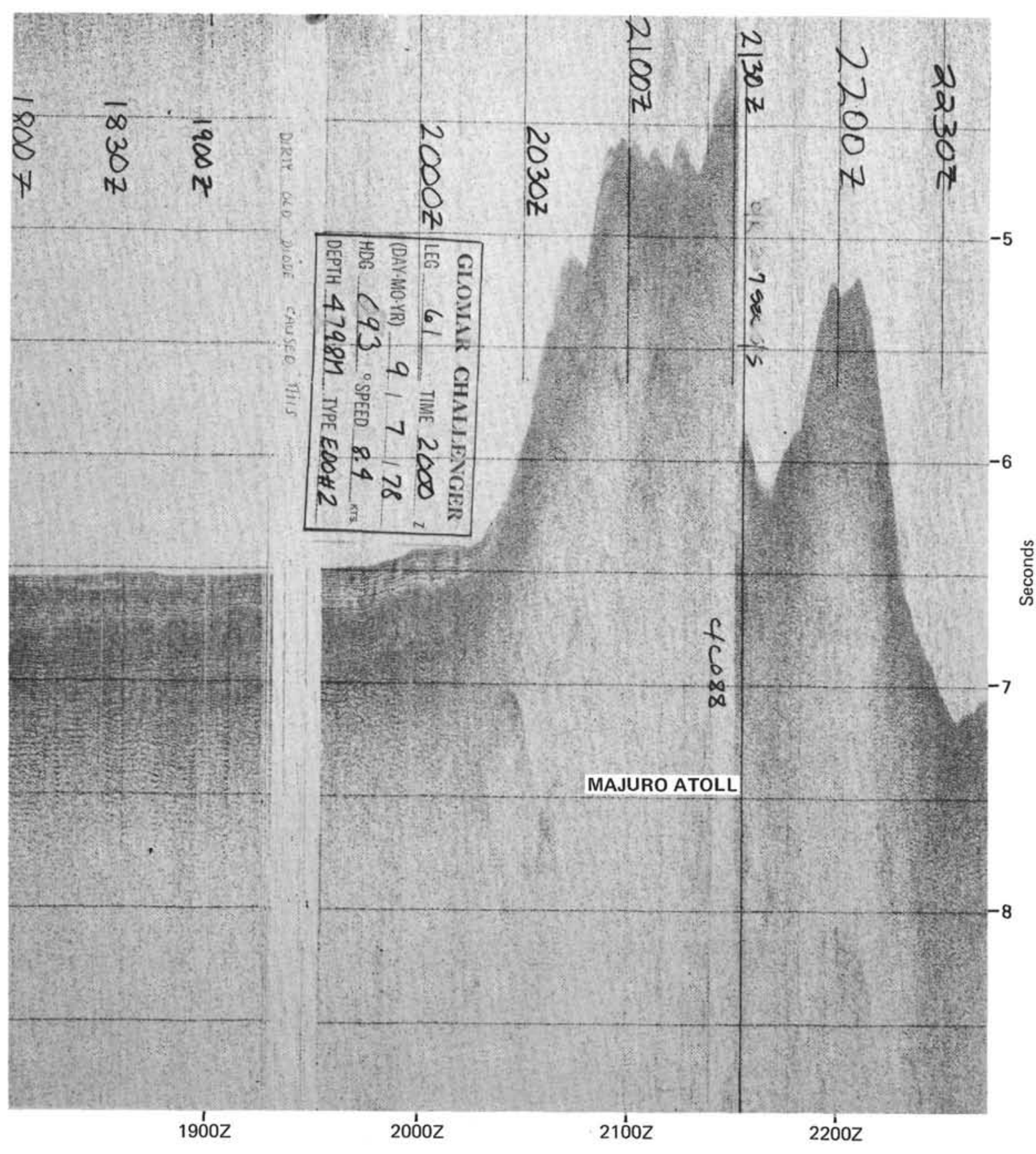




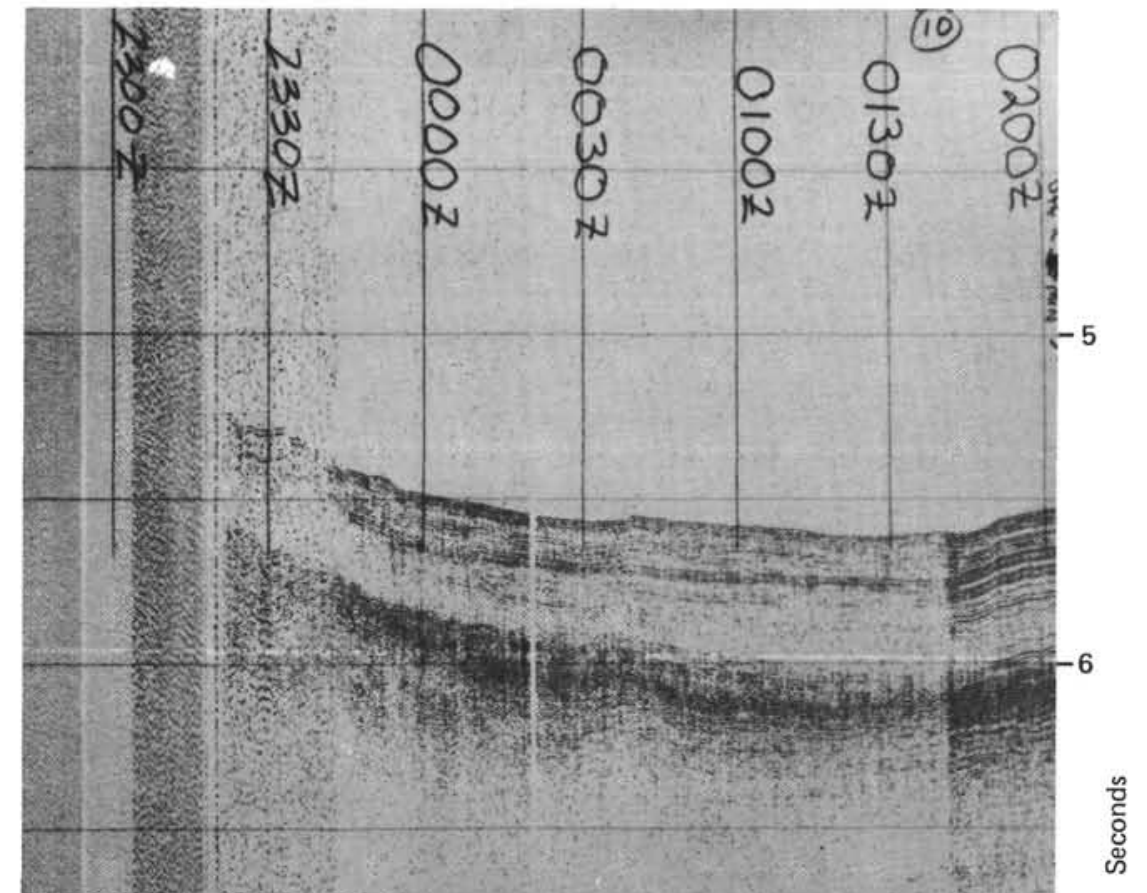

LEAVING MAJURO ATOLL

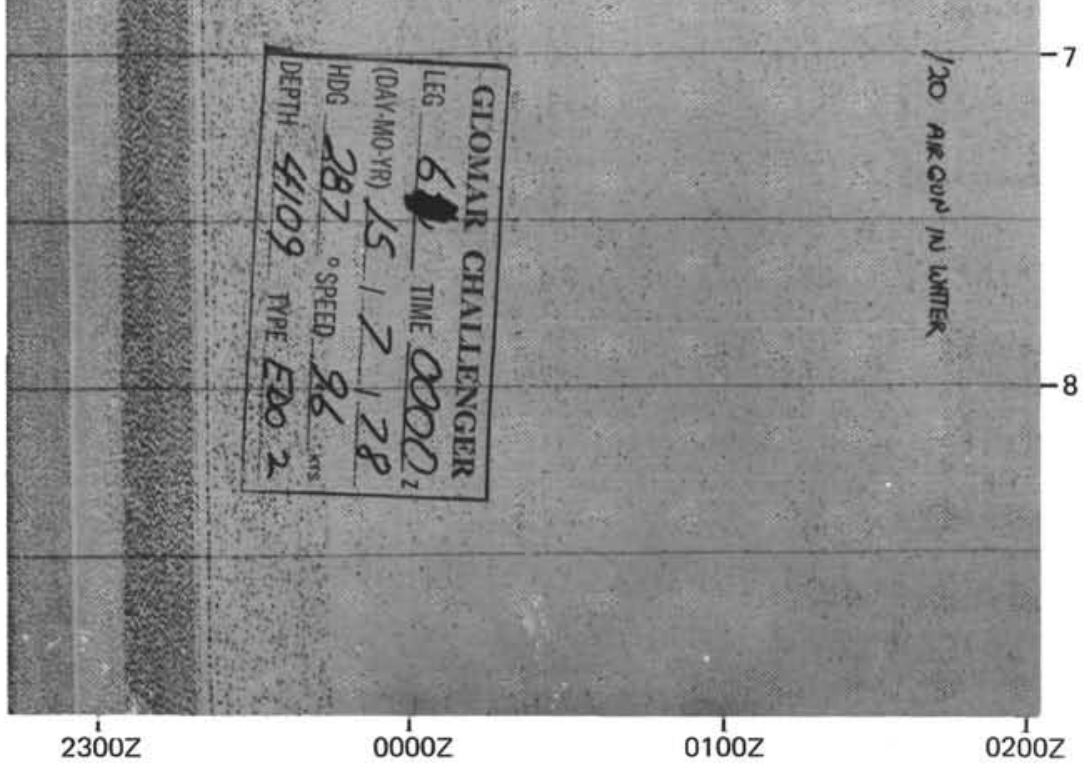

Figure 3. (Continued). 


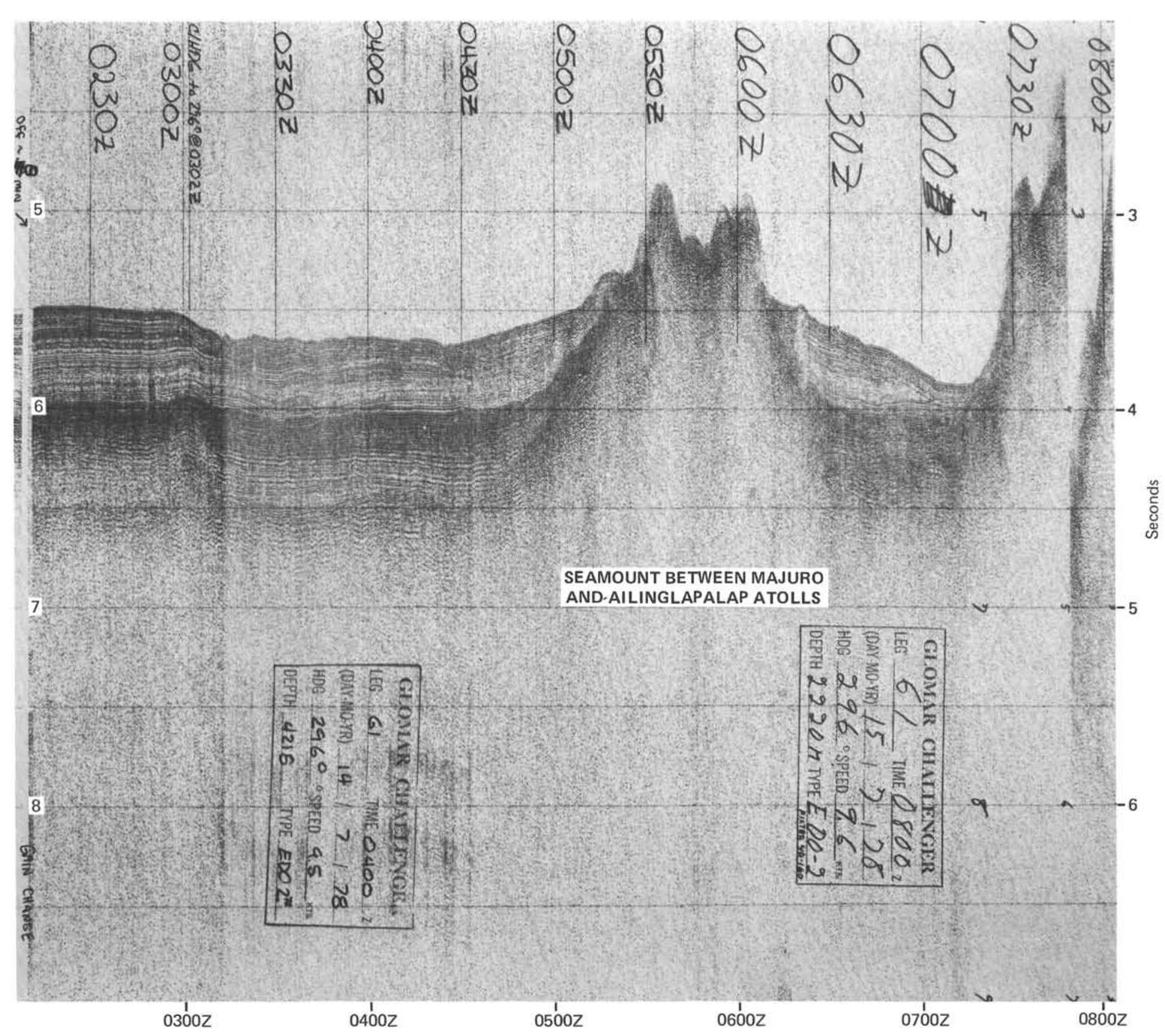




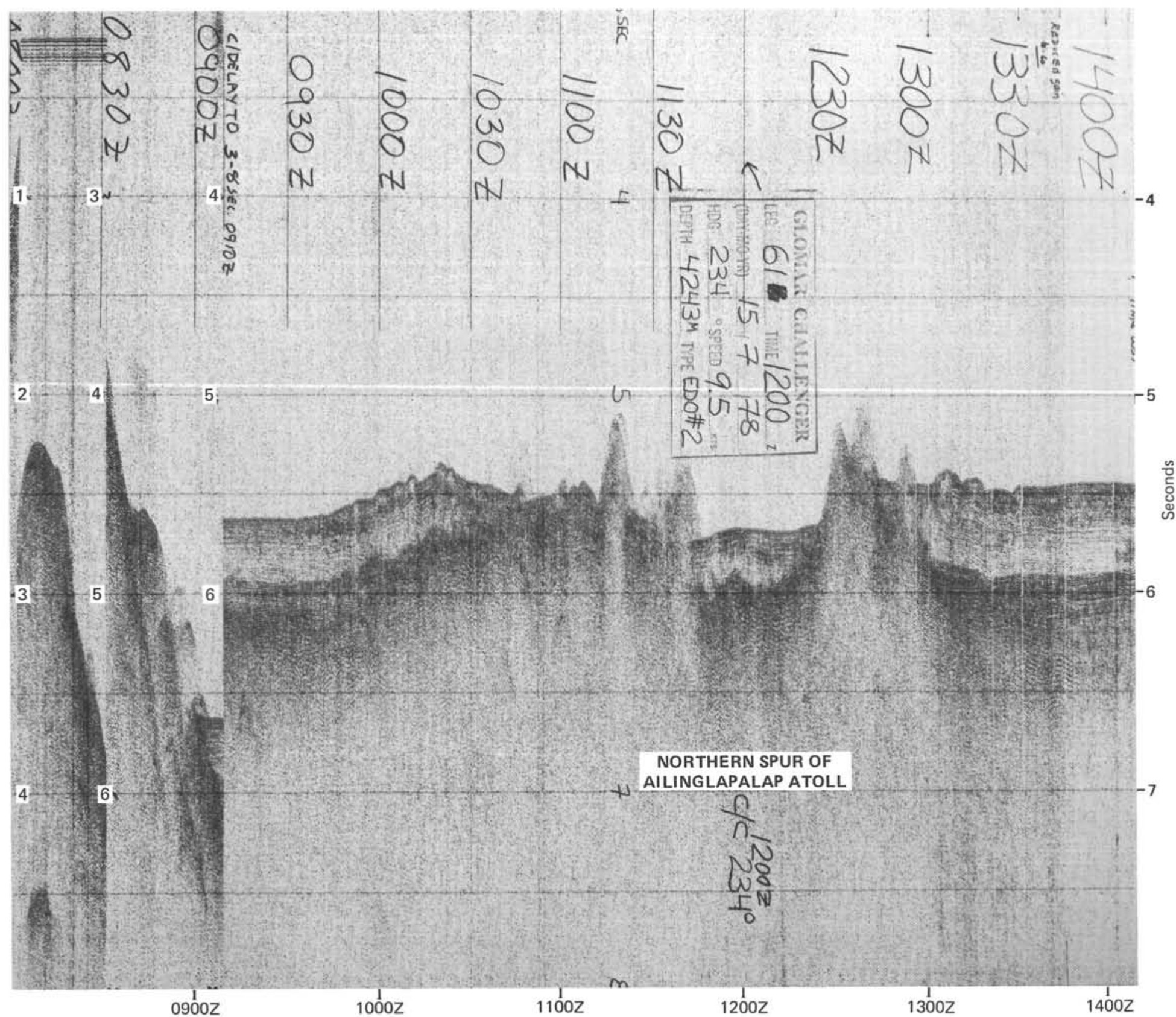




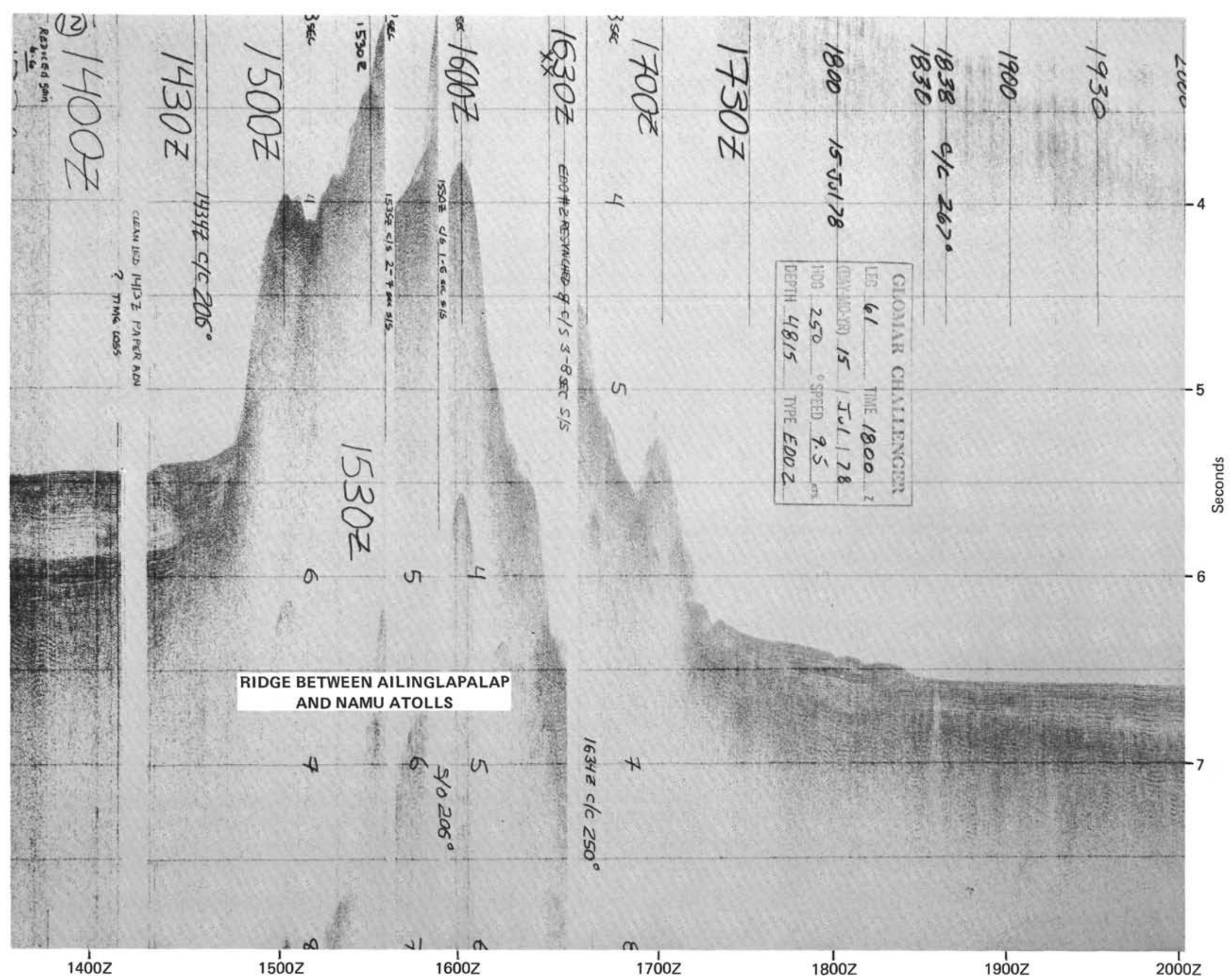




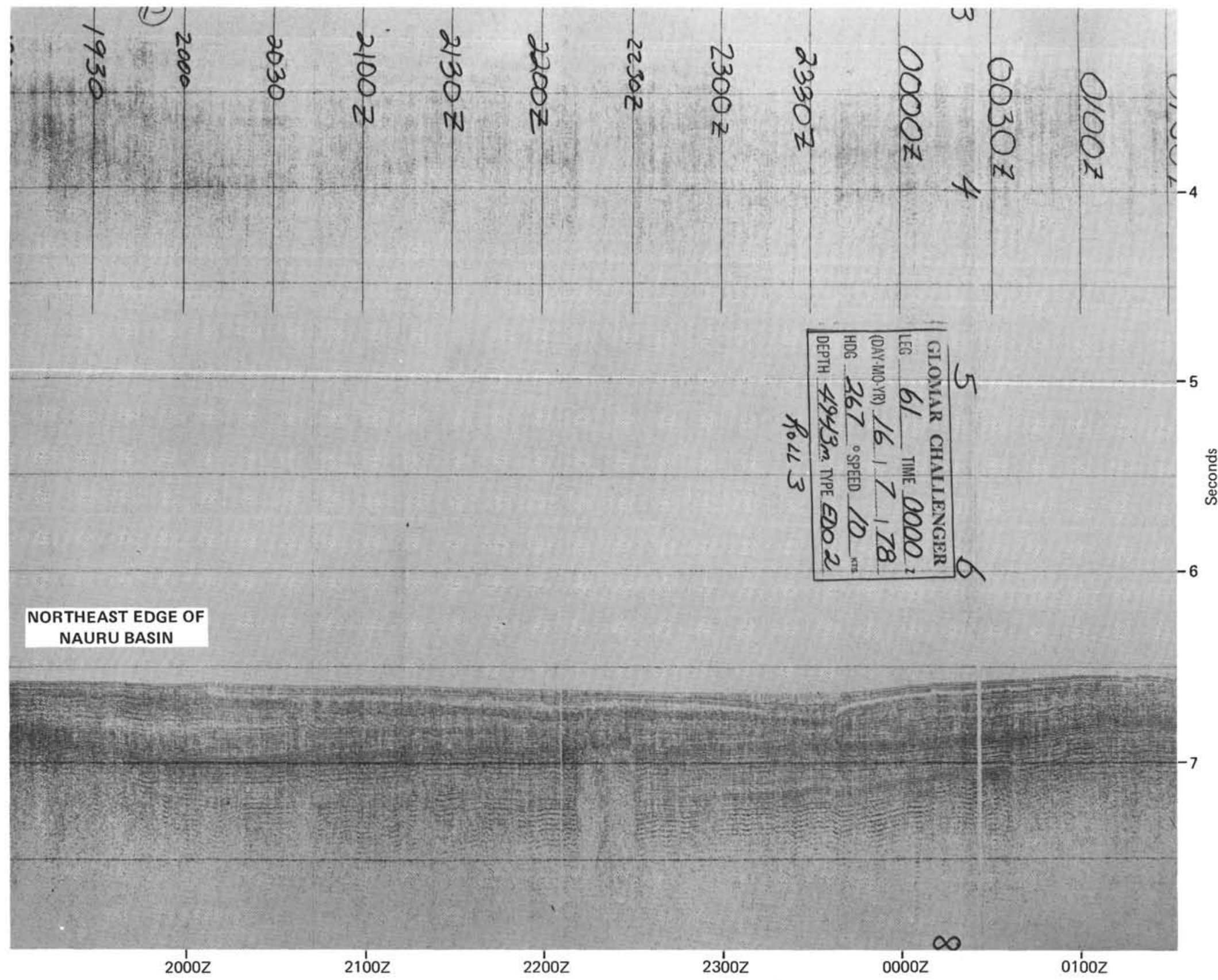




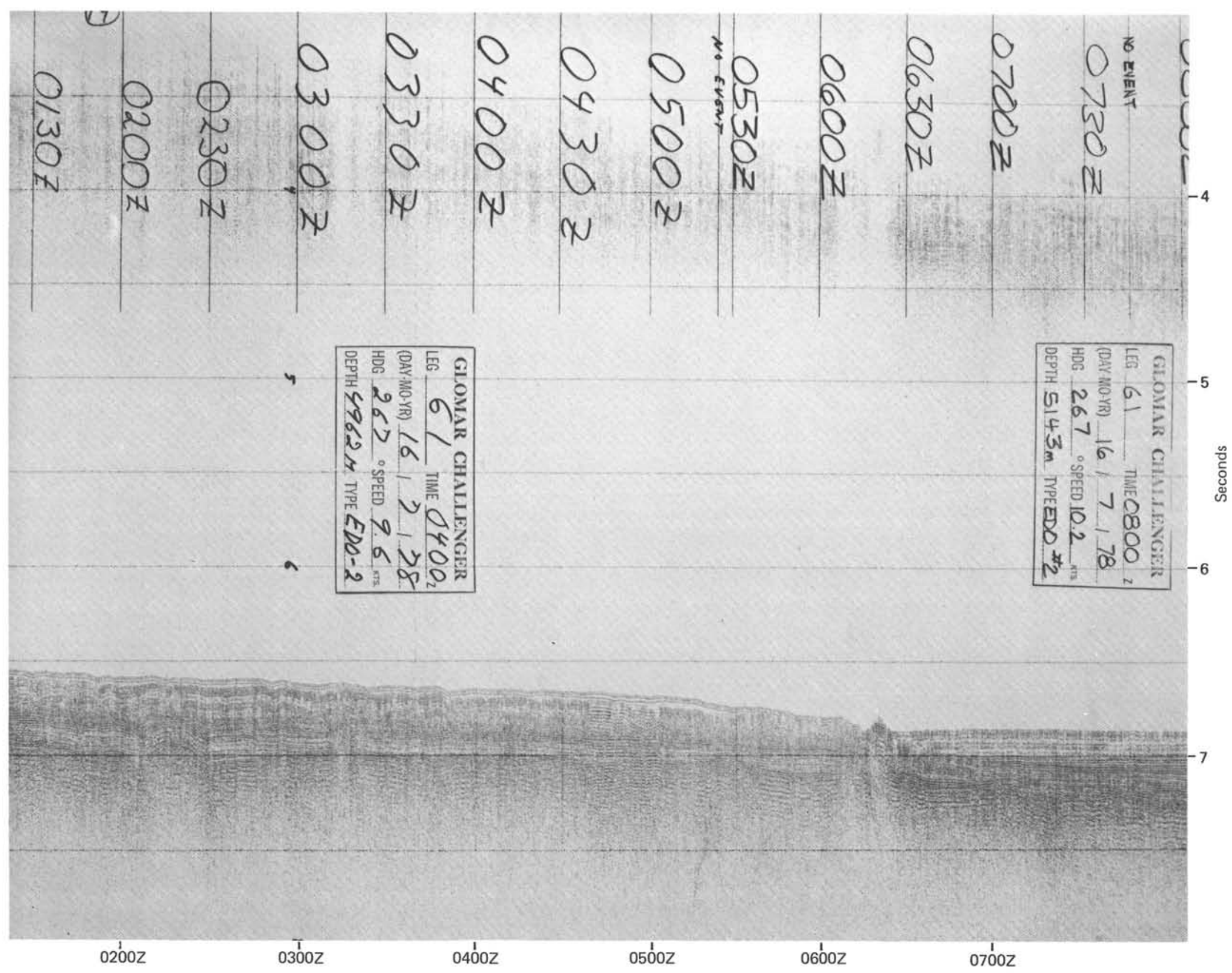




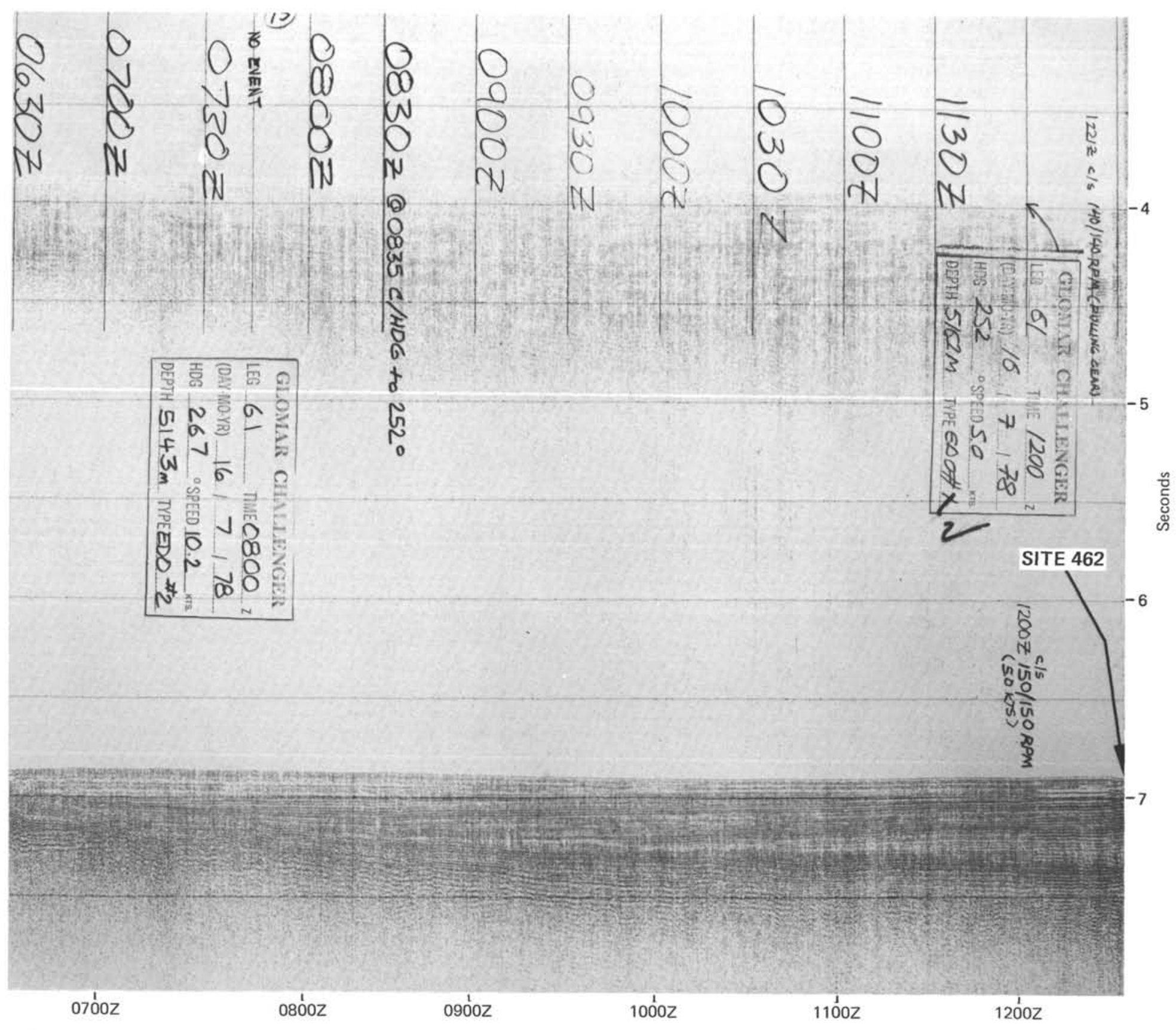

